\title{
Variety
Trials
}

\section{Evaluation of Winegrapes in British Columbia: New Cultivars and Selections from Germany and Hungary}

\author{
Andrew G. Reynolds ${ }^{1}$,
} Margaret Cliff ${ }^{1}$, Douglas A. Wardle ${ }^{2}$, and
Marjorie King

AdDitional InDex words. Vitis, breeding, clone

Summary. Eighty-five cultivars, selections and clones of winegrapes (Vitis) from European breeding and selection programs were evaluated between 1993-95 in a randomized completeblock experiment. These included selections from Alzey, Freiburg, Geilweilerhof, Geisenheim, Weinsberg, and Würzburg (Germany); Hungary; and the former USSR. Vines were grown under an organic management regime that included sodium silicate

$\overline{\text { Agriculture and Agri-Food Canada, Summerland, }}$ B.C. V0H $1 \mathrm{ZO}$.

Acknowledgments: Field and Lab: Rochelle Eisen, field/ lab technician; Martin Drew, assistant. Winemaking: Doug Wardle, technical assistant; Deepank Utkhede, summer student. Tasting: Mike Bouthillier, Margaret Cliff, Tony Cottrell, Pascal Delaquis, Marj King, Benoit Girard, Hilary Graham, Linda Herbert, Andy Reynolds, Laszlo Veto, Doug Wardle, Jim Wild.

${ }^{1,2}$ Research scientist and technician, respectively. Present address of senior author: Cool Climate Oenology \& Viticulture Institute, Brock University, St. Catharines, ON L2S 3Al, Canada. sprays for powdery mildew (Uncinula necator) control and oil + detergent for insect control but with little to no nitrogen or other nutritional inputs. The Weinsberg cultivars Heroldrebe and Helfensteiner showed promise viticulturally and sensorially as alternatives to 'Pinot noir'. Cultivars from Geisenheim ('Gm 7117-10' and 'Gm 7117-26') and Würzburg ('Cantaro' and 'Fontanara') appeared promising as 'Riesling' alternatives; many displayed similar sensory characteristics to 'Riesling', along with reasonable viticultural performance. Cultivars selected at Alzey ('Faberrebe'), Freiburg ('Nobling'), and Weinsberg ('Holder') displayed sensory characteristics superior to the standard cultivar Müller-Thurgau, with very intense muscat, pear, fig, and spicy aromas and flavors. Several muscat-flavored Hungarian white wine cultivars appeared to be superior viticulturally and sensorially to the standard 'Csabagyongye'; these included 'Kozma Palne Muscotaly', 'Zefir', and 'Zengo'. Miscellaneous red wine cultivars that showed promise included Geilweilerhof cultivar Regent, and Hungarian selections Kozma 55 and Kozma 525. Vine yields decreased substantially in the 3-year evaluation period, primarily due to lack of nitrogen. Many of these cultivars appeared to be highly adaptable to viticultural regions where cold winters and low heat units during fruit maturation presently restrict cultivar choices.

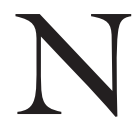
umerous grape breeding programs exist throughout the world with objectives of introducing wine grape cultivars with improved adaptabilities to climatic adversities and pests. Many of the programs in Europe have focused on interspecific crosses involving Vitis vinifera that combine high wine quality with resistance to diseases. Two wellknown interspecific cultivars from the breeding program at Geilweilerhof, Germany, include 'Castor' and 'Pollux' [('Oberlin 595') $F_{1} \times$ 'Foster's White Seedling'; Alleweldt, 1980]. Many intraspecific $V$. vinifera winegrape cultivars and selections have also been bred to add to the cultivar diversity within various winegrowing regions of the world. Examples of these include 'Ehrenfelser' ('Riesling' X 'Sylvaner', 1929) from the breeding program in Geisenheim, Germany, and 'Scheurebe' ('Riesling' x 'Sylvaner', 1911) from the breeding station at Alzey, Germany (Becker 1984). As an adjunct to these programs, ongoing clonal selection programs for $V$. viniferawinegrapes focus upon cultivar improvement through selection of superior clones. 'Riesling', 'Chardonnay', and 'Pinot noir' are three cultivars from which several unique clones have been selected and named (Becker, 1984; Bernard and Leguay, 1984; Boidron, 1995).

As new vineyard regions are being established throughout North America, grapegrowers are increasingly demanding more choices in planting material. Frequently, expanding viticultural regions, such as the midwestern United States and northern Virginia (Wolf and Miller, 2001; Wolf and Warren, 2000), have adverse climatic conditions, primarily fluctuating winter temperatures, that prevent success of traditional $V$. vinifera winegrape cultivars. In the coastal regions of the Pacific Northwest, lack of heat units during fruit maturation is usually the mitigating factor. Little or no information is available from these regions documenting the performance of recently introduced European varieties, selections, and clones in specific regions of North America. Three notable exceptions are the clonal trials on 'Pinot noir' in Oregon (Price and Watson, 1995; Price et al., 1988; Watson et al., 1988) and New York (Pool et al., 1995), and those in California on numerous cultivars including 'Cabernet Sauvignon', 'Chardonnay', 'Merlot', and 'Zinfandel' (Bettiga, 1995; Wolpert, 1995).

Hundreds of interspecific hybrids, intraspecific $V$. vinifera cultivars, and clones of $V$. vinifera winegrapes have been bred or selected throughout Europe. Many of these have been selected for specific environmental conditions 
and have had no evaluation under North American conditions. At the same time, many viticultural regions outside the traditional wine producing areas have become established throughout the United States and Canada, and these industries have created a demand for new varieties that are adaptable to the unique climatic conditions present in these regions. The objective of this study was to evaluate a large collection of winegrape cultivars and selections (both interspecific and intraspecific $V$. vinifera) in terms of field performance, fruit composition, and wine sensory attributes. Relative performances of 'Chardonnay' and 'Pinot noir' clones are described in a related publication (Reynolds, 1996a). Moreover, because a growing organic movement has been evident in British Columbia since the late 1980s, this trial was also regarded as an opportunity to gain some experience with organic viticulture across a wide range of cultivars. The latter approach was made possible and is considered justified because no downy mildew (Plasmopara viticola), black rot (Guignardia bidwellii), phomopsis leaf blight (Phomopsis viticola), or eutypa dieback (Eutypa lata) occur in the region.

\section{Materials and methods}

ENVIRONMENTAL CONDITIONS. Cultivars were evaluated at the Pacific Agri-Food Research Centre, Summerland, B.C., Canada. The site was situated in the Okanagan Valley, which is considered an arid continental climate. Elevation was $454 \mathrm{~m}$ (1488ft) above sea level and latitude was $49^{\circ} 34^{\prime} \mathrm{N}$. Mean annual precipitation for the test period was $287.8 \mathrm{~mm}$ (11.33 inches), of which $165.6 \mathrm{~mm}$ (6.52 inches) fell during the April to October growing season. Mean temperature of the warmest month (July) was $20.8{ }^{\circ} \mathrm{C}$ $\left(69.44{ }^{\circ} \mathrm{F}\right)$; mean minimum winter temperature was $-6.4^{\circ} \mathrm{C}\left(20.48{ }^{\circ} \mathrm{F}\right)$; absolute minimum winter temperature was $-15.7{ }^{\circ} \mathrm{C}\left(3.74{ }^{\circ} \mathrm{F}\right)$. Growing degree days (GDD; $10{ }^{\circ} \mathrm{C}$ base) were $1142.9\left(2089.2 \mathrm{GDD}\right.$ using a $50^{\circ} \mathrm{F}$ base) (Drought and Stretch, 1996).

EXPERIMENTAL DESIGN AND PLANT MATERIAL. All plant materials were obtained as rooted cuttings via the Centre for Plant Health, Saanichton, B.C. Incidence of phylloxera in the region is very low, and therefore own-rooted grapevines were not at risk of phylloxera injury. Soil type at the planting site was
Skaha gravelly sandy loam (Kelley and Spilsbury, 1949) with an organic matter content of $1.9 \%$ and a $\mathrm{pH}$ of 7.8 [British Columbia Ministry of Agriculture, Fisheries and Food (BCMAFF), 1992]. Vines were planted in May 1988 at $1.8 \mathrm{~m} \times 2.7 \mathrm{~m}(6 \mathrm{ft} \times 9 \mathrm{ft}$; vine $\times$ row), trained to $0.6-\mathrm{m}-$ ( 24 inches) high bilateral cordons, and pruned to enough two-node spurs to provide 15 shoots $/ \mathrm{m}$ row $(4.6$ shoots $/ \mathrm{ft}$ ). Shoots were trained upwards in a vertical trellis and were trimmed at the tops around mid-July (berries $<$ pea size). Vines were irrigated throughout the life of the planting by drippers spaced $1.0 \mathrm{~m}$ (40 inches) apart and delivering 4.5 $\mathrm{L} \cdot \mathrm{h}^{-1}(1.19 \mathrm{gal} / \mathrm{h})$. Irrigation began each season after the emergence of three to five leaves, and was operated until the last cultivars were harvested in late October. Water was applied for $2 \mathrm{~h}$ per day [14.4 L/vine (3.79 gal/ vine) per day or $30,549 \mathrm{~L} \cdot \mathrm{ha}^{-1}(3266$ gal/acre) per day].

Pest control was done according to local recommendations (BCMAFF, 1996) from 1988-92 and organically thereafter. During 1993-95, powdery mildew was controlled by 1000 $\mathrm{mg} \cdot \mathrm{L}^{-1}(\mathrm{ppm})$ sodium silicate applied five times between late May and early September. Research with potassium silicate, ongoing at the time of this trial, demonstrated its effectiveness as a grape powdery mildew prophylactic (Reynolds et al., 1996). Leafhoppers were controlled using two applications of $0.5 \% \mathrm{v} / \mathrm{v}$ (dormant oil $)+$ (Tide) detergent (Proctor and Gamble, Toronto) during early summer. This was adapted from a combination of local practice within the organic community as well as encouraging results with plant-derived oils on grape powdery mildew and botrytis bunch rot control (Reynolds, 1996b). Thrips populations were suppressed by these measures, but not adequately. According to the Certified Organic Association of British Columbia (COABC) (2003), sodium silicate is an allowable product and dormant oil is a regulated item, but detergents are presently prohibited; use of Tide was therefore the only noncertified product used during the course of the trial. No nitrogen was applied during 1993-95 due to the nonavailability of sufficient quantities of any allowable certified organic source, such as: composted animal manures; green manures; miscellaneous composted organic materials; blood, vegetable, or hoof and horn meals; fish meals and emulsions (COABC, 2003). Application of blood meal was attempted through the irrigation system, but this was unsuccessful (and hence abandoned) due to clogging of drippers.

Experimental design was a randomized complete block with 85 cultivars, selections and clones, four blocks, and three-vine treatment replicates. To facilitate data analysis, a series of sub-experiments were designated for the following groups of cultivars, selections, and/or clones, organized in terms of the country or experiment station of origin: 1) Alzey cultivars and selections;2) Freiburg and Weinsberg cultivars and selections; 3 ) Geilweilerhof cultivars and selections; 4) Geisenheim cultivars and selections; 5) Würzburg cultivars and selections; 6) Hungarian muscat cultivars and selections; 7) Hungarian white wine cultivars and selections; 8) miscellaneous red wine varieties and selections. The complete list of cultivars, selections, and clones evaluated in this trial, along with their origins (Alleweldt and Dettweiler-Münch, 1992; Hillebrand et al., 1984), sorted according to the 1993 harvest dates, is found in Table 1. Performances of the 'Chardonnay' and 'Pinot noir' clones are described elsewhere (Reynolds, 1996a).

YIELD COMPONENTS. Harvest date was based on a 15-point scoresheet (Table 1) used to assess fruit maturity. Cultivars were sampled every $48 \mathrm{~h}$ and harvested once the fruit maturity index reached between 12 and 15 . Yield per vine was recorded at harvest. A random 100-berry sample was taken from each treatment replicate to determine berry weight and chemical composition.

BERRY CHEMICAL COMPOSITION. Berry samples were juiced, and soluble solids concentration (SSC) and $\mathrm{pH}$ were measured thereon by a temperature-compensated Abbé refractometer (AO Scientific, Buffalo, N.Y.) and Fisher Accumet $825 \mathrm{MP} \mathrm{pH}$ meter (Fisher Scientific, Vancouver, B.C.), respectively. Titratable acidity (TA) was measured with a Brinkmann automatic titrating ensemble (Metrohm, Herisau, Switzerland) and expressed as tartaric acid equivalents (Amerine and Ough, 1980).

WINEMAKING: MUST AND WIND COMPOSITION; SENSORY ANALYSIS. Winemaking. Based on preliminary data collected during a pilot project 
Table 1. Harvest dates (listed sequentially for 1993) and origins for 79 winegrape cultivars evaluated at Summerland, B.C., in 1993-95.

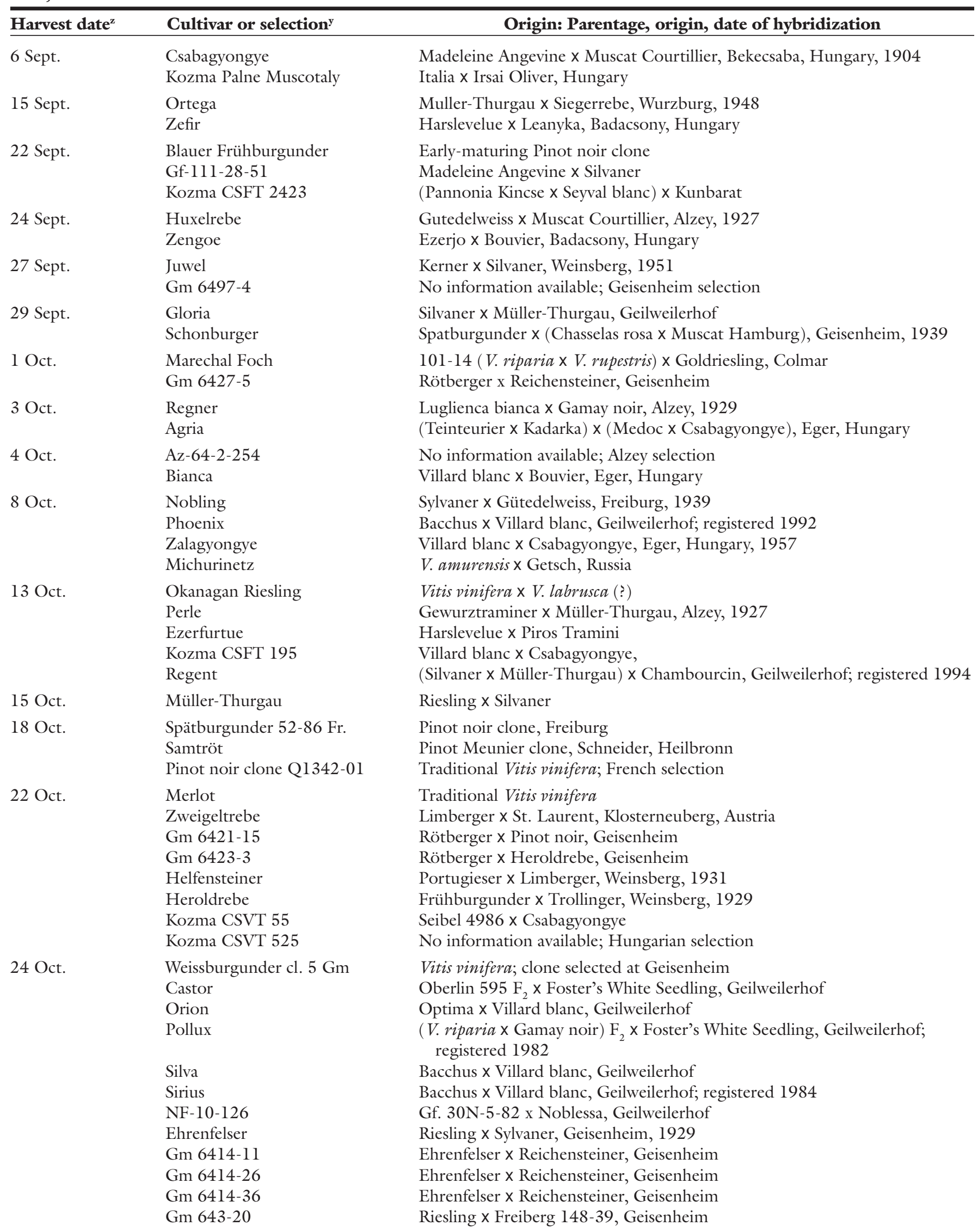

Table 1 continued on next page. 
Table 1. Continued.

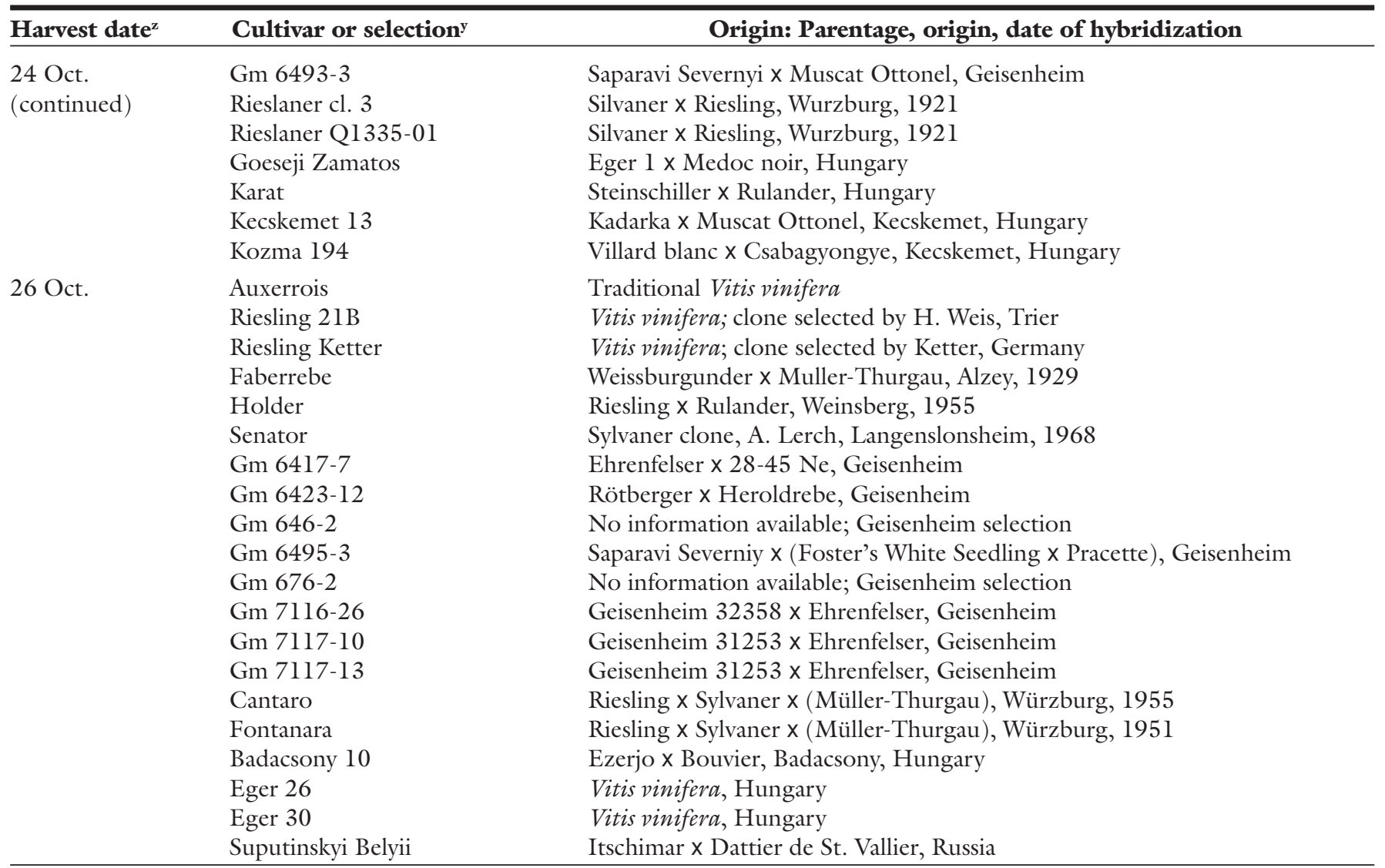

${ }^{2}$ Harvest was based upon a 15-point subjective scale that encompassed color ( $0-2$ points), ease of removal of berries from the pedicels ( $0-2$ points), texture upon touch ( 0 -1 point), texture upon initial bite ( $0-2$ points), mechanical features of the pulp ( $0-2$ points), aroma ( $0-2$ points), flavor upon chewing ( $0-2$ points), flavor release from the skin ( $0-1$ point), and aftertaste ( $0-1$ point).

yall vines were own-rooted.

in 1991 (BCMAFF 1992, 1993), 36 cultivars, selections and clones were chosen for winemaking in 1993. All fruit from each treatment replicate was retained from these cultivars at harvest in 1993 for winemaking. Cultivars not chosen for winemaking were those previously identified by members of the local industry as having undesirable sensory characteristics, or those not substantially different from cultivars already being grown (BCMAFF, 1992, 1993). Grapes were typically stored at $2{ }^{\circ} \mathrm{C}\left(35.6^{\circ} \mathrm{F}\right)$ for $24 \mathrm{~h}$, crushed in a Garolla crusher-destemmer, and the crushed grapes from each treatment replicate were collected in 20-L (5.3 gal) plastic pails. White wine grapes were pressed in a rack-and-cloth press $24 \mathrm{~h}$ after crushing, sulfited to $25 \mathrm{mg} \cdot \mathrm{L}^{-1}$ free sulfur dioxide $\left(\mathrm{SO}_{2}\right)$, settled at $2{ }^{\circ} \mathrm{C}$ for $24 \mathrm{~h}$, and racked into 20-L glass carboys. Musts were inoculated with VLl (Saccharomyces cerevisiae; Lallemand Corp., Montreal) yeast. Fermentations were carried out to dryness at $15{ }^{\circ} \mathrm{C}\left(59.0^{\circ} \mathrm{F}\right)$. Fruit from each red wine grape treatment replicate were crushed into individual 20 - L fermenters, sulfited to $25 \mathrm{mg} \cdot \mathrm{L}^{-1}$ free $\mathrm{SO}_{2}$, and separate primary fermentations were carried out using ECI 118 yeast on each treatment replicate for 6 $\mathrm{d}$ at $30^{\circ} \mathrm{C}\left(86.0^{\circ} \mathrm{F}\right)$, after which the essentially dry wines were pressed. Caps were submerged two to three times daily by punching down. Fermentation was completed in glass carboys at $20^{\circ} \mathrm{C}$ $\left(68.0^{\circ} \mathrm{F}\right)$. At the completion of fermentation, all wines were racked, sulfited to $50 \mathrm{mg} \cdot \mathrm{L}^{-1} \mathrm{SO}_{2}$, cold stabilized for $21 \mathrm{~d}$ at $-2^{\circ} \mathrm{C}\left(28.4^{\circ} \mathrm{F}\right)$, and stored at $1^{\circ} \mathrm{C}\left(33.8^{\circ} \mathrm{F}\right)$ until bottling. Before bottling, fermentations from adjacent field blocks (blocks $1+2$; blocks $3+4$ ) were blended together in equal volumes to provide two composites for tasting. Wines were thereafter filtered through a $0.45-\mu \mathrm{m}$ pad and a $0.22-\mu \mathrm{m}$ cartridge prior to bottling.

Must and wine composition. A $250-\mathrm{mL}(8.5 \mathrm{fl} \mathrm{oz})$ must sample was collected from all white wine replicates immediately after pressing, and from all red wine replicates after crushing. Similarly, 250-mL samples were taken from all wines prior to bottling; an additional set of samples was also taken from the red wines after pressing. Soluble solids concentration of the must, and $\mathrm{TA}$ and $\mathrm{pH}$ of the musts and wines were determined as previously described for the berry samples. Anthocyanins in the red wines were measured by the $\mathrm{pH}$ shift method (Amerine and Ough, 1980). Ethanol concentration in the wines was determined using a Hewlett-Packard 5700 gas chromatograph (Hewlett-Packard Canada, Mississauga, Ont.).

Sensoryanalysis. Sensory analyses were performed on the wines during Apr. to June 1997 after 36 months of bottle storage at $11{ }^{\circ} \mathrm{C}\left(51.8^{\circ} \mathrm{F}\right)$ by a 12 -member panel with prior wine evaluation experience and documented skills (based on $50 \%$ correct responses in triangle tests in several other related experiments). Aroma descriptors and retronasal aroma/tactile (flavor) descriptors were chosen by consensus following evaluation of numerous aroma and flavor samples (Table 2 ) and examples of wines produced from the 
Table 2. Descriptors and standards used for sensory analysis of wines produced from German and Hungarian winegrape cultivars and selections at Summerland, B.C., in 1993.

\begin{tabular}{|c|c|}
\hline Name of descriptor & Details of reference preparation \\
\hline & Pinot noir alternatives ${ }^{z}$ \\
\hline Cherry & $\begin{array}{l}1 \mathrm{~mL} \text { each of natural cherry extract R-10242y } \\
+ \text { imitation black cherry extract F- } 6534^{y}\end{array}$ \\
\hline Berry & $\begin{array}{l}1 \mathrm{~mL} \text { each of these extracts }{ }^{\mathrm{y}} \text { : imitation black raspberry F-3365, } \\
\text { imitation strawberry F-5662, and natural + imitation } \\
\text { raspberry R-895 }\end{array}$ \\
\hline Currant & $250 \mathrm{~mL} \operatorname{syrup}^{\mathrm{x}}$ \\
\hline Plum/prune & $350 \mathrm{~mL} 1981$ experimental golden plum wine \\
\hline Grassy/hay & 300 mL 1993 experimental Semillon wine \\
\hline Vegetal/bell pepper & $1 \mathrm{~mL}$ vegetable extract $2 \mathrm{M}-44444^{\mathrm{v}}$ \\
\hline Black pepper/spicy & $1 \mathrm{~mL}$ pepper extract $7407676^{\mathrm{y}}$ \\
\hline Licorice/anise & $1 \mathrm{~mL}$ anise seed oil $1751^{\mathrm{y}}$ \\
\hline Caramel/candy & $250 \mathrm{~mL}$ Andres medium dry Canadian sherry \\
\hline
\end{tabular}

Riesling and alternatives; Müller-Thurgau and alternatives; Hungarian muscats" Floral

Lychee

Citrus

Vegetal

Peach/apricot

Cedar/pine

Melon

Spicy

$1 \mathrm{~mL}$ linaloo

$100 \mathrm{~mL}$ canned lychee syrup

$0.25 \mathrm{~mL}$ each of lemon $531^{\mathrm{y}}$, orange $138216^{\mathrm{x}}$, grapefruit $138214^{\mathrm{u}}$, and lime $138212^{\mathrm{u}}$

$0.25 \mathrm{~g}$ each of asparagus, celery, and cauliflower powders ${ }^{\mathrm{t}}$

$50 \mathrm{~mL}$ apricot syrup ${ }^{\mathrm{x}}$

$0.5 \mathrm{~mL}$ spruce beer $4691^{y}$

Half of one honeydew melon, cubed, steeped for $20 \mathrm{~min}$

2 drops each of clove $1435^{y}$, nutmeg $1427^{y}$, spice blend TN-151-3y, and cinnamon oils

\section{Miscellaneous reds}

Berry

$1 \mathrm{~mL}$ each of these extracts ${ }^{\mathrm{y}}$ : imitation black raspberry F-3365, imitation strawberry F-5662, and natural + imitation raspberry R-895

Currant

Plum/prune

Vegetal

Earthy

$50 \mathrm{~mL}$ syrup

$1 \mathrm{~mL}$ date type extract SPL $3333^{y}$

$250 \mathrm{~mL} 1993$ experimental Semillon wine

$1 \mathrm{~mL}$ imitation mushroom extract F-6327:

${ }^{2}$ Quantities indicated were added to $1 \mathrm{~L}(33.8 \mathrm{fl} \mathrm{oz})$ of experimental 1987 Gamay noir wine. $1.00 \mathrm{~mL}=0.034$

$\mathrm{fl} \mathrm{oz} ; 1.00 \mathrm{~g}=0.035 \mathrm{oz}$.

Givaudin Ltd., Atlanta, Ga.

xSummerland Sweets Ltd., Summerland, B.C.

"Quantities indicated were added to $0.5 \mathrm{~L}(16.9 \mathrm{fl} \mathrm{oz})$ neutral white wine.

'Quest International Nederland BV, Naarden, The Netherlands.

"Alex Fries, Inc., Cincinatti, Ohio.

tFIS (Food Information Service), University of British Columbia, Vancouver, B.C.

${ }^{s}$ Quantities indicated were added to $0.5 \mathrm{~L}$ of 1993 experimental Chancellor wine.

project. Descriptive analysis entailed $50-\mathrm{mL}$ (1.7 fl oz) samples of each treatment presented randomly under white light in clear, tulip-shaped glasses. Panelists were initially requested to assess several aroma terms, after which the samples were removed, their numerical codes changed, and their order re-randomized for flavor assessment. The two tasting replicates were evaluated for each cultivar in separate sessions. A 100-mm- (3.9 inches) line scoresheet anchored at 0 (low) and extending to 100 (high) was used to quantify intensity of each aroma and flavor descriptor.
Statistical analysis. All data were analyzed by cultivar within each group using SAS (SAS Institute, Cary, N.C.). The General Linear Models Procedure was utilized for analysis of variance with cultivar $\times$ block used as an error term. Sensory data were subjected to both analysis of variance and principal components analysis (PCA). PCA is a multivariate statistical technique that uses correlation coefficients to elucidate the nature of shared variability among several measured chemical and/or sensory variables (Noble, 1988). Intercorrelated variables, or factors, are then identified from the data set. The group of factors that are responsible for the greatest degree of variability in the data set are referred to as principal component 1 (PCl). Most of the variability is usually explained in the first three PCs that are identified; the first two PCs are typically portrayed as a two-dimensional diagram with $\mathrm{PCl}$ represented on the horizontal axis and PC2 on the vertical axis. Each sensory variable is depicted graphically as a vector (eigenvector) originating at the axis of PC1 and PC2; the length of each eigenvector is relative to the degree to which that variable contributes to the overall variability in the data set. Eigenvectors that are parallel to each other are considered highly correlated; those $180^{\circ}$ from each other are inversely correlated. The position of each sample relative to the eigenvectors is reflective of their general sensory profile. The distance of each sample relative to the axis of $\mathrm{PCl}$ and $\mathrm{PC} 2$ is proportional to the intensity of the sample. The SAS Means, Correlation, Factor Data, and Score Procedures were used for PCA.

\section{Results and discussion}

YIELD AND BERRY COMPOSITION. Alzey cultivars. Standard 'MüllerThurgau' outyielded the four cultivars from the Alzey breeding program (Table 3). 'Huxelrebe' consistently had highest berry weight (Table 3 ). 'Faberrebe' had highest SSC and also highest TA in 1994-95 (Table 3). Soluble solids in both 'Faberrebe' and 'AZ 64-2-254' were above 26\% SSC in 1994-95, but those of other cultivars were considerably lower, and each season most were within the $18 \%$ to $24 \%$ SSC range commonly considered desirable for winemaking (Table 3). TA values were also within the $5-10 \mathrm{~g} \cdot \mathrm{L}^{-1}$ (5000-10,000 ppm) range considered desirable for winemaking (Table 3 ). No pattern was detected among the cultivars with respect to $\mathrm{pH}$; all were within the 3.0 to 3.5 range considered desirable for winemaking (Table 3 ).

Freiburg and Weinsberg cultivars. Standard cultivar Müller-Thurgau tended to outyield the white wine cultivars from Freiburg and Weinsberg, with the exception of 'Juwel' (Table 3 ). 'Nobling' consistently had highest berry weights, while 'Holder' had lowest berry weights in 2 of 3 years (Table 3). 'Nobling' also had highest SSC in 2 of 3 years, and was the only white wine cultivar in this group that 
Table 3. Yield and berry composition of several winegrape cultivars and selections from European breeding programs, evaluated at Summerland, B.C., in 1993-95. Cultivars in bold type within each table subsection are commercial standards.

\begin{tabular}{|c|c|c|c|c|c|c|c|c|c|c|c|c|c|c|c|}
\hline \multirow{2}{*}{$\begin{array}{l}\text { Origin and } \\
\text { cultivar }\end{array}$} & \multicolumn{3}{|c|}{ Yield $\left(t \cdot h a^{-1}\right)^{z}$} & \multicolumn{3}{|c|}{ Berry wt $(g)^{z}$} & \multicolumn{3}{|c|}{ Soluble solids (\%) } & \multicolumn{3}{|c|}{$\begin{array}{c}\text { Titratable } \\
\text { acidity }\left(\mathrm{g} \cdot \mathrm{L}^{-1}\right)^{\mathrm{z}}\end{array}$} & \multicolumn{3}{|c|}{ pH } \\
\hline & 1993 & 1994 & 1995 & 1993 & 1994 & 1995 & 1993 & 1994 & 1995 & 1993 & 1994 & 1995 & 1993 & 1994 & 1995 \\
\hline \multicolumn{16}{|c|}{ White winegrape cultivars originating from Landessanstalt fur Rebenzuchtung, Alzey, Germany (Rbeinbessen) } \\
\hline Müller-Thurgau & 17.2 & $10.2 \mathrm{a}$ & $3.6 \mathrm{a}$ & $1.34 \mathrm{ab}$ & $1.10 \mathrm{~b}$ & $1.36 \mathrm{~b}$ & $17.8 \mathrm{bc}$ & $21.9 \mathrm{~d}$ & $23.0 \mathrm{e}$ & $7.3 \mathrm{c}$ & $7.7 \mathrm{~b}$ & $5.4 \mathrm{~d}$ & $3.14 \mathrm{~b}$ & $3.16 \mathrm{c}$ & $3.13 \mathrm{e}$ \\
\hline Faberrebe & 6.8 & $2.2 \mathrm{c}$ & $1.6 \mathrm{ab}$ & $1.23 \mathrm{bc}$ & $0.88 \mathrm{e}$ & $1.14 \mathrm{c}$ & $22.8 \mathrm{a}$ & $27.4 \mathrm{a}$ & $26.5 a$ & $7.8 \mathrm{bc}$ & $9.0 \mathrm{a}$ & $7.9 \mathrm{a}$ & $3.29 \mathrm{a}$ & $3.01 \mathrm{~d}$ & $3.39 b$ \\
\hline Huxelrebe & 2.0 & $6.9 \mathrm{~b}$ & $1.3 \mathrm{ab}$ & $1.58 \mathrm{a}$ & $1.70 \mathrm{a}$ & $1.60 \mathrm{a}$ & $17.0 \mathrm{c}$ & $19.8 \mathrm{e}$ & $24.8 \mathrm{~d}$ & $8.6 \mathrm{~b}$ & $7.6 \mathrm{~b}$ & $7.4 b$ & $3.15 b$ & $3.14 \mathrm{c}$ & $3.31 \mathrm{~d}$ \\
\hline Significance & NS & $* *$ & * & $* *$ & $* * *$ & $* * *$ & $* * *$ & $* * *$ & $* * *$ & ** & $* * *$ & $* * *$ & $* * *$ & $* * *$ & $* * *$ \\
\hline
\end{tabular}

Cultivars originating from Staatliches Weibauinstitut, Freiburg $\left.{ }^{F}\right)$ \& Staatliche Lebr-und Versuchsanstalt, Weinsberg ( $\left.{ }^{W}\right)$, Germany (Baden-Würtemmburg)

\begin{tabular}{|c|c|c|c|c|c|c|c|c|c|c|c|c|c|c|c|}
\hline Müller-Thurgau & $17.2 \mathrm{a}$ & $10.2 \mathrm{a}$ & $3.6 \mathrm{ab}$ & $1.34 \mathrm{bc}$ & $1.10 \mathrm{c}$ & $1.36 \mathrm{~b}$ & $17.8 \mathrm{c}$ & $21.9 \mathrm{~d}$ & $23.0 \mathrm{~d}$ & $7.3 \mathrm{c}$ & $7.7 \mathrm{c}$ & $5.4 \mathrm{~d}$ & $3.14 b$ & $3.16 \mathrm{a}$ & $3.13 \mathrm{c}$ \\
\hline Juwel $^{\mathrm{W}}$ & $11.4 \mathrm{ab}$ & $5.9 \mathrm{ab}$ & $5.0 \mathrm{a}$ & $1.36 \mathrm{bc}$ & $1.19 \mathrm{~b}$ & $1.01 \mathrm{~d}$ & $19.4 \mathrm{~b}$ & $22.9 c$ & $24.8 \mathrm{a}$ & $9.2 \mathrm{a}$ & $8.2 b$ & $8.6 \mathrm{~b}$ & $2.88 \mathrm{c}$ & $3.07 \mathrm{c}$ & $3.13 \mathrm{c}$ \\
\hline Nobling ${ }^{\mathrm{F}}$ & $6.2 \mathrm{~b}$ & $4.0 \mathrm{~b}$ & $1.5 \mathrm{c}$ & $1.63 \mathrm{ab}$ & $1.37 \mathrm{a}$ & $1.50 \mathrm{a}$ & $22.7 \mathrm{a}$ & $29.5 a$ & $24.4 \mathrm{~b}$ & $8.5 b$ & $7.1 \mathrm{~d}$ & $9.0 \mathrm{a}$ & $3.14 b$ & $3.14 b$ & $3.21 \mathrm{~b}$ \\
\hline $\begin{array}{l}\text { Significance } \\
\text { (white grapes) }\end{array}$ & * & * & * & $* * *$ & $* * *$ & * * & ** & $* * *$ & * & * & $* * *$ & $* * *$ & $* * *$ & $* * *$ & $* * *$ \\
\hline Spätburgunder & & & & & & & & & & & & & & & \\
\hline $52-86 \mathrm{Fr}$ & 10.6 & 4.1 & $6.1 \mathrm{~A}$ & $1.13 \mathrm{~B}$ & $0.84 \mathrm{C}$ & $0.86 \mathrm{C}$ & $20.0 \mathrm{~A}$ & $27.8 \mathrm{~A}$ & $25.8 \mathrm{~A}$ & $8.8 \mathrm{~A}$ & $7.2 \mathrm{C}$ & $8.2 \mathrm{~B}$ & $3.15 \mathrm{~B}$ & $3.27 \mathrm{~A}$ & $3.19 \mathrm{~B}$ \\
\hline Helfensteiner ${ }^{W}$ & 13.7 & 6.2 & $2.7 \mathrm{~B}$ & $1.91 \mathrm{~A}$ & $1.91 \mathrm{~A}$ & $1.98 \mathrm{~A}$ & $19.0 \mathrm{~B}$ & $21.6 \mathrm{C}$ & $21.7 \mathrm{C}$ & $7.9 \mathrm{~A}$ & $7.9 \mathrm{~B}$ & $8.5 \mathrm{~A}$ & $3.09 \mathrm{~B}$ & $3.15 \mathrm{C}$ & $3.11 \mathrm{C}$ \\
\hline
\end{tabular}

Significance

(red grapes)

Cultivars originating from Bundesforschungsanstalt für Rebenzüchtung Geilweilerhof, Siebeldingen, Germany (Rbeinpfalz)

Riesling clone

Müller-Thurg

Castor (B-7-2)

Gloria

Orion clone 6

(GA 58-30)

Phoenix

(GA 49-22)

Pollux

(B-6-18)

Silva clone 5

(GA 54-14)

Sirius (GA 51-27)

Gf 111-28-51

NF 10-126

Significance

Riesling clone

21B Weis

Senator

Gm 6414-11

Gm 6414-26

Gm 6414-36

Gm 6417-7

Gm 6423-12

Gm 6427-5

Gm 643-20

Gm 646-2

Gm 6493-3

Gm 6495-3

Gm 6497-4

Gm 676-2

Gm 7116-26

Gm 7117-10

Gm 7117-13

Significance

(white grapes) 9.8b-e $\quad 2.8 \mathrm{~d}$

$17.1 \mathrm{ab} \quad 10.2 \mathrm{bc}$

$15.2 \mathrm{abc} 12.6 \mathrm{~b}$

$13.4 \mathrm{bc} \quad 8.0 \mathrm{c}$

$2.2 \mathrm{bcd} \quad 1.14 \mathrm{de}$

$3.6 \mathrm{bcd} \quad 1.34 \mathrm{~d}$

$5.9 \mathrm{ab} \quad 1.79 \mathrm{bc}$

$\begin{array}{lll}0.88 \mathrm{i} & 0.95 \mathrm{i} & 17.7 \mathrm{f}\end{array}$

$21.9 \mathrm{~g} \quad 26.1 \mathrm{c} \quad 10.5 \mathrm{a}$

$9.2 \mathrm{bc} \quad 9.4 \mathrm{a} \quad 3.01 \mathrm{f}$

$2.91 \mathrm{i} \quad 3.15 \mathrm{i}$

$\begin{array}{lllll}1.24 \mathrm{f} & 1.47 \mathrm{~d} & 18.4 \mathrm{ef} & 23.2 \mathrm{e} & 22.6 \mathrm{j}\end{array}$

$7.3 \mathrm{~d} \quad 7.7 \mathrm{e}$

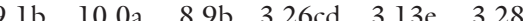

\section{$9.5 \mathrm{~b}-\mathrm{e} \quad 4.4 \mathrm{~cd} \quad 3.3 \mathrm{bcd} \quad 1.31 \mathrm{~d}$}

$1.12 \mathrm{~g} \quad 1.27 \mathrm{f} \quad 21.0 \mathrm{a}-\mathrm{d} \quad 23.5 \mathrm{~d} \quad 26.6 \mathrm{a}$

$6.6 \mathrm{def} \quad 6.8$

$13.0 \mathrm{bc} \quad-\mathrm{y}^{\mathrm{y}} \quad 5.5 \mathrm{abc} \quad 2.06 \mathrm{a}$

--- $\quad 1.81 \mathrm{c} \quad 19.2 \mathrm{cde}$

$6.3 \mathrm{ef}$

9.7

$17.6 \mathrm{a}$

$8.7 \mathrm{a} \quad 1.74 \mathrm{bc}$

$1.68 \mathrm{~b} \quad 1.81 \mathrm{c}$

$19.5 \mathrm{def}$

$---\quad 21.4 \mathrm{k} \quad 5.9 \mathrm{f}$

--- $\quad 6.4 \mathrm{~h} \quad 3.32 \mathrm{bc}$

$8.2 \mathrm{c} \quad 3.31 \mathrm{bc}$

$12.5 \mathrm{bcd} \quad 5.9 \mathrm{~cd} \quad 3.6 \mathrm{bcd} \quad 1.85 \mathrm{~b}$

$\begin{array}{lllll}1.58 \mathrm{c} & 1.81 \mathrm{c} & 21.6 \mathrm{abc} & 22.3 \mathrm{f} & 23.6 \mathrm{~g}\end{array}$

$\begin{array}{lllll}1.50 \mathrm{e} & 1.83 \mathrm{~b} & 20.8 \mathrm{bcd} & 23.7 \mathrm{c} & 23.8 \mathrm{f}\end{array}$

$\begin{array}{lllll}1.53 \mathrm{~d} & 1.24 \mathrm{~g} & 22.0 \mathrm{a} & 20.4 \mathrm{~h} & 24.8 \mathrm{e}\end{array}$

$4.1 \mathrm{e} \quad 5.4 \mathrm{~cd} \quad 4.1 \mathrm{bcd} \quad 1.57 \mathrm{c}$

$4.6 \mathrm{de} 5.3 \mathrm{~cd}$ l.1d $0.99 \mathrm{e}$

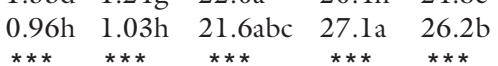

$\begin{array}{llllll}6.9 \mathrm{de} & 7.6 \mathrm{e} & 7.4 \mathrm{f} & 3.38 \mathrm{~b} & 3.25 \mathrm{~b} & 3.34 \mathrm{~b}\end{array}$ $\begin{array}{lllllll}7.2 \mathrm{~d} & 8.7 \mathrm{~d} & 8.2 \mathrm{c} & 3.17 \mathrm{de} & 3.04 \mathrm{~g} & 3.16 \mathrm{~h}\end{array}$ $\begin{array}{llllll}3.8 \mathrm{~g} & 4.7 \mathrm{~h} & 3.1 \mathrm{j} & 3.65 \mathrm{a} & 3.4 \mathrm{la} & 3.7 \mathrm{la}\end{array}$ $6.5 \mathrm{def} \quad 8.7 \mathrm{~d} \quad 7.5 \mathrm{e} \quad 3.25 \mathrm{~cd} \quad 3.07 \mathrm{f} \quad 3.31 \mathrm{~d}$ Cultivars originating from Institut fur Rebenzuchtung, Geisenbeim, Germany (Rbeingau)

\begin{tabular}{|c|c|c|c|c|c|c|c|c|c|c|c|c|c|c|}
\hline $9.8 c-f$ & $8 d-g$ & $2 \mathrm{~cd}$ & $f g$ & $8 \mathrm{i}$ & $5 p$ & $.7 \mathrm{gh}$ & $.9 q$ & & b & $9.2 \mathrm{c}$ & $9.4 \mathrm{c}$ & 3.0lhij & $\ln$ & $5 j$ \\
\hline $1.0 \mathrm{~g}$ & $2.0 \mathrm{fg}$ & $6.1 \mathrm{a}$ & & $23 \mathrm{~d}$ & 020 & $29.4 \mathrm{a}$ & 328 & $23.2 \mathrm{n}$ & $2 \mathrm{ijk}$ & $4.2 \mathrm{q}$ & $6.7 \mathrm{n}$ & $3.61 \mathrm{a}$ & $3.64 \mathrm{a}$ & $45 a$ \\
\hline $12.4 \mathrm{a}-\mathrm{f}$ & $5.2 \mathrm{a}-\mathrm{f}$ & $1.3 \mathrm{~d}$ & tdef & 0.831 & $1.30 \mathrm{~g}$ & $18.9 \mathrm{~d}-\mathrm{h}$ & $6.5 \mathrm{~h}$ & $23.8 \mathrm{k}$ & $7 \mathrm{hij}$ & $5.4 \mathrm{p}$ & $6.9 \mathrm{~m}$ & 99ef & $3 \mathrm{e}$ & $33 b$ \\
\hline $5.9 \mathrm{fg}$ & $2.3 \mathrm{efg}$ & $2.8 \mathrm{bcd}$ & $.22 \mathrm{~d}-\mathrm{g}$ & 0.831 & $1.31 \mathrm{f}$ & $20.4 c-f$ & & $.3 \mathrm{~h}$ & 7.9hij & $8.5 \mathrm{~g}$ & $7.4 \mathrm{k}$ & $0 \mathrm{e}$ & $14 \mathrm{~d}$ & $4 \mathrm{~d}$ \\
\hline $8.6 \mathrm{def}$ & $.0 \mathrm{c}-\mathrm{g}$ & $0^{3-9}$ & & 0.831 & $.36 c$ & & $9 \mathrm{k}$ & & $8.1 \mathrm{f}-\mathrm{i}$ & & $7.6 \mathrm{j}$ & & & \\
\hline $3.9 \mathrm{a}-\mathrm{e}$ & $.3 \mathrm{~b}-\mathrm{g}$ & $16 d$ & & & & & & & & & & & & \\
\hline $1-d$ & $.5 \mathrm{a}-\mathrm{f}$ & $3.2 \mathrm{a}-\mathrm{d}$ & & 1.3 & e & $\mathrm{d}$ & $2 c$ & $27.6 \mathrm{a}$ & 9. & 6 & $7.9 \mathrm{~g}$ & $g-j$ & 1 & $7 \mathrm{i}$ \\
\hline$-f$ & $.9 \mathrm{ab}$ & 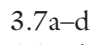 & & $\mathrm{b}$ & b & & o & & 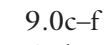 & 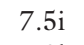 & $7.8 \mathrm{~h}$ & & $8 \mathrm{~g}$ & $4 \mathrm{k}$ \\
\hline $0 \mathrm{ab}$ & $6 a-f$ & & & & 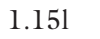 & & $8 q$ & $6 q$ & 6. & 7.01 & $7 \mathrm{i}$ & & $4 c$ & \\
\hline $6.4 \mathrm{abc}$ & $a-f$ & & & $0.92 \mathrm{~g}$ & $.05 n$ & & $26.7 \mathrm{~g}$ & & 9. & $0.7 \mathrm{~b}$ & $7.9 \mathrm{~g}$ & & $4 \mathrm{~m}$ & 21 \\
\hline 12.1a-f & $2.3 \mathrm{efg}$ & $5.7 \mathrm{ab}$ & def & $1.02 \mathrm{f}$ & $1.24 \mathrm{j}$ & 17.8 & $26.4 \mathrm{i}$ & $21.9 p$ & 7.9hij & $7.4 \mathrm{j}$ & $8.1 \mathrm{f}$ & & $2.97 \mathrm{k}$ & $2 p$ \\
\hline $17.9 \mathrm{a}$ & $.2 \mathrm{a}$ & $3.6 a-d$ & & $1.55 \mathrm{a}$ & $1.63 a$ & & $28.2 \mathrm{~d}$ & 22.80 & $8.2 \mathrm{fgh}$ & 8. & $8.2 \mathrm{e}$ & & $3.04 \mathrm{~h}$ & $99 n$ \\
\hline - & c & & & & h & & 23 & & & & $5.9 \mathrm{o}$ & & $\mathrm{lb}$ & \\
\hline & $.9 \mathrm{a}-\mathrm{e}$ & $2.1 \mathrm{~cd}$ & & & $1.10 \mathrm{~N}$ & & $27.7 \mathrm{e}$ & & & & & & & \\
\hline $12.8 \mathrm{a}-\mathrm{f}$ & $2 a b c$ & $5.2 \mathrm{abc}$ & & $0.82 \mathrm{~m}$ & $1.11 \mathrm{~m}$ & & & $25.1 \mathrm{~d}$ & $8.3 \mathrm{e}-\mathrm{h}$ & 6.50 & $7.5 \mathrm{k}$ & & $3.00 \mathrm{j}$ & $0 \mathrm{~g}$ \\
\hline $8.8 \mathrm{def}$ & $5 a-d$ & $3.0 \mathrm{a}-\mathrm{d}$ & & $0.84 \mathrm{k}$ & $1.33 \mathrm{~d}$ & 20.9b-e & $27.3 \mathrm{f}$ & $24.4 \mathrm{~g}$ & $10.9 \mathrm{ab}$ & $10.9 \mathrm{a}$ & $10.4 \mathrm{a}$ & $2.99 \mathrm{j}$ & $2.87 \mathrm{o}$ & 260 \\
\hline $5.9 \mathrm{fg}$ & $1.1 \mathrm{~g}$ & $1.3 \mathrm{~d}$ & $1.21 \mathrm{~d}-\mathrm{g}$ & $0.88 \mathrm{i}$ & $1.18 \mathrm{k}$ & $20.0 \mathrm{c}-\mathrm{g}$ & $30.5 b$ & $23.9 \mathrm{j}$ & $11.7 \mathrm{a}$ & $8.9 \mathrm{~d}$ & $9.7 \mathrm{~b}$ & $2.99 \mathrm{j}$ & $3.14 \mathrm{~d}$ & $3.02 \mathrm{~m}$ \\
\hline
\end{tabular}

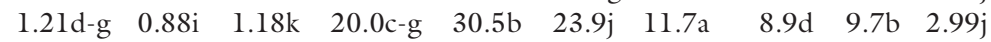

$3.14 \mathrm{~d} \quad 3.02 \mathrm{~m}$

Table 3 continued on next page. 
Table 3. Continued.

\begin{tabular}{|c|c|c|c|c|c|c|c|c|c|c|c|c|c|c|c|}
\hline \multirow{2}{*}{$\begin{array}{l}\text { Origin and } \\
\text { cultivar }\end{array}$} & \multicolumn{3}{|c|}{ Yield $\left(t \cdot h a^{-1}\right)^{z}$} & \multicolumn{3}{|c|}{ Berry wt $(g)^{z}$} & \multicolumn{3}{|c|}{ Soluble solids (\%) } & \multicolumn{3}{|c|}{$\begin{array}{c}\text { Titratable } \\
\text { acidity }\left(g \cdot L^{-1}\right)^{z}\end{array}$} & \multicolumn{3}{|c|}{ pH } \\
\hline & 1993 & 1994 & 1995 & 1993 & 1994 & 1995 & 1993 & 1994 & 1995 & 1993 & 1994 & 1995 & 1993 & 1994 & 1995 \\
\hline Q1342-01 & $6.7 \mathrm{~B}$ & $6.3 \mathrm{~A}$ & $0.5 \mathrm{C}$ & $1.09 \mathrm{~B}$ & $0.86 \mathrm{C}$ & $1.04 \mathrm{C}$ & $23.4 \mathrm{~A}$ & $27.0 \mathrm{~A}$ & $25.0 \mathrm{~B}$ & $7.5 \mathrm{~A}$ & $7.1 \mathrm{~A}$ & $6.6 \mathrm{~B}$ & $3.29 \mathrm{~A}$ & $3.25 \mathrm{~A}$ & $3.33 \mathrm{~B}$ \\
\hline Gm 6421-15 & $17.3 \mathrm{~A}$ & $5.8 \mathrm{AB}$ & $1.6 \mathrm{~B}$ & $1.47 \mathrm{~A}$ & $1.24 \mathrm{~A}$ & $1.58 \mathrm{~A}$ & $18.1 \mathrm{~B}$ & $23.3 \mathrm{C}$ & $25.6 \mathrm{~A}$ & 7.1AB & $6.3 \mathrm{~B}$ & $7.6 \mathrm{~A}$ & $3.23 \mathrm{~B}$ & $3.19 \mathrm{~B}$ & $.29 \mathrm{C}$ \\
\hline Gm 6423-3 & $12.4 \mathrm{AB}$ & $3.4 \mathrm{~B}$ & $2.9 \mathrm{~A}$ & $1.31 \mathrm{AB}$ & $1.08 \mathrm{~B}$ & $1.40 \mathrm{~B}$ & $16.9 \mathrm{C}$ & $25.7 \mathrm{~B}$ & $24.5 \mathrm{C}$ & $6.4 \mathrm{~B}$ & $5.7 \mathrm{C}$ & $6.6 \mathrm{~B}$ & $3.21 \mathrm{~B}$ & $3.25 \mathrm{~A}$ & $3.40 \mathrm{~A}$ \\
\hline $\begin{array}{l}\text { Significance } \\
\text { (red grapes) }\end{array}$ & \multicolumn{14}{|c|}{$\begin{array}{c}\text { Cultivars originating from Bayerische Landesanstalt fur Wein-, Obst- und Gartenbau, } \\
\text { Würzburg-Veitshochbeim, Germany (Franken) }\end{array}$} & \\
\hline \multicolumn{16}{|l|}{ Riesling clone } \\
\hline $21 B$ & 9.8 & $2.8 b$ & 2.2 & $1.14 \mathrm{~b}$ & $0.88 \mathrm{c}$ & $0.95 c$ & $17.7 \mathrm{c}$ & $21.9 \mathrm{~d}$ & $26.1 \mathrm{c}$ & $10.5 \mathrm{a}$ & $9.2 \mathrm{a}$ & $9.4 \mathrm{a}$ & $3.01 \mathrm{e}$ & $2.91 \mathrm{~d}$ & $3.15 \mathrm{~d}$ \\
\hline Cantaro & 8.3 & $3.1 \mathrm{~b}$ & 2.8 & $1.01 \mathrm{~b}$ & $0.93 c$ & $1.03 \mathrm{c}$ & $24.0 \mathrm{a}$ & $29.3 \mathrm{a}$ & $25.9 b$ & $8.0 \mathrm{c}$ & $7.5 \mathrm{c}$ & $7.6 \mathrm{~b}$ & $3.22 b c$ & $3.06 \mathrm{a}$ & $3.17 \mathrm{~d}$ \\
\hline Fontanara & 9.8 & $3.4 \mathrm{~b}$ & 1.7 & $1.05 \mathrm{~b}$ & $1.08 \mathrm{~b}$ & $1.19 \mathrm{~b}$ & $20.4 \mathrm{ab}$ & $27.6 c$ & $25.5 \mathrm{~d}$ & $7.9 \mathrm{c}$ & $8.8 \mathrm{~b}$ & $6.0 \mathrm{c}$ & $3.18 \mathrm{~cd}$ & $3.04 \mathrm{~b}$ & $3.31 \mathrm{~b}$ \\
\hline 01 & 10.0 & $---y$ & $---y$ & $0.99 \mathrm{~b}$ & --- & --- & $20.3 \mathrm{ab}$ & --- & --- & $9.3 \mathrm{~b}$ & --- & --- & $3.15 \mathrm{~cd}$ & --- & --- \\
\hline Signific & NS & *** & NS & $* * *$ & *** & $* * *$ & * & *** & $* * *$ & *** & *** & $* * *$ & *** & $* * *$ & *** \\
\hline \multicolumn{16}{|c|}{ Hungarian muscat-flavored winegrape cultivars } \\
\hline $\begin{array}{l}\text { Csabagyongye } \\
\text { Goecseji }\end{array}$ & $4.8 \mathrm{c}$ & $---y$ & $---y$ & $1.48 \mathrm{~b}$ & $1.96 \mathrm{~b}$ & --- & $18.9 \mathrm{a}$ & $21.5 \mathrm{~d}$ & --- & $7.2 \mathrm{~d}$ & $6.2 \mathrm{e}$ & --- & $3.29 \mathrm{a}$ & $3.46 \mathrm{a}$ & --- \\
\hline $\begin{array}{c}\text { Zamatos } \\
\text { Kozma Palne }\end{array}$ & $4.8 \mathrm{c}$ & $7.5 a$ & 3.1 & $1.47 \mathrm{~b}$ & $1.22 \mathrm{~d}$ & $1.19 \mathrm{c}$ & $21.1 \mathrm{a}$ & $21.7 \mathrm{c}$ & $24.4 \mathrm{~d}$ & $9.7 \mathrm{~b}$ & $9.7 \mathrm{~b}$ & $10.5 \mathrm{a}$ & $3.10 \mathrm{~b}$ & $3.05 \mathrm{~d}$ & $3.16 \mathrm{c}$ \\
\hline Muscotaly & $2 \mathrm{ab}$ & $4.7 \mathrm{~b}$ & $--^{y}$ & $2.11 \mathrm{a}$ & $2.30 \mathrm{a}$ & $1.81 \mathrm{a}$ & $16.4 \mathrm{~b}$ & 17. & $27.6 \mathrm{a}$ & $6.9 \mathrm{~d}$ & $6.6 \mathrm{c}$ & $4.0 \mathrm{~d}$ & $3.26 \mathrm{a}$ & $3.32 b$ & $3.69 \mathrm{a}$ \\
\hline Zefir & $8.1 \mathrm{bc}$ & $4.8 \mathrm{~b}$ & 2.1 & $1.21 \mathrm{c}$ & 1.04 & $1.04 \mathrm{~d}$ & $21.2 \mathrm{a}$ & 23 & $27.0 \mathrm{~b}$ & $8.5 \mathrm{c}$ & $6.4 \mathrm{~d}$ & $4.8 \mathrm{~b}$ & 3. & $3.20 \mathrm{c}$ & $3.63 b$ \\
\hline & $12.4 \mathrm{a}$ & $7.6 a$ & 3.7 & $1.65 b$ & $1.73 \mathrm{c}$ & $1.54 \mathrm{~b}$ & $19.1 \mathrm{a}$ & $22.3 b$ & $26.7 \mathrm{c}$ & $11.4 \mathrm{a}$ & $11.4 \mathrm{a}$ & $8.7 \mathrm{c}$ & 2.5 & $2.83 \mathrm{e}$ & $3.09 \mathrm{~d}$ \\
\hline Significance & ** & ** & NS & $* * *$ & $* * *$ & $* * *$ & $* *$ & $* * *$ & $* * *$ & $* * *$ & $* * *$ & $* * *$ & $* * *$ & $* * *$ & $* * *$ \\
\hline Kecskemet 13 & $3.1 \mathrm{~d}$ & $2.0 \mathrm{bc}$ & $1.8 \mathrm{bc}$ & $1.51 b c$ & 1.3 & $1.41 \mathrm{~d}$ & $20.2 \mathrm{de}$ & $25.3 \mathrm{e}$ & $21.6 \mathrm{k}$ & $8.5 \mathrm{de}$ & $6.1 \mathrm{j}$ & $9.3 \mathrm{c}$ & 3.1 & $3.17 \mathrm{c}$ & $3.16 \mathrm{~h}$ \\
\hline Kozma 195 & $13.7 \mathrm{a}$ & $6.5 \mathrm{ab}$ & $6.1 \mathrm{a}$ & $1.69 \mathrm{ab}$ & $1.60 \mathrm{~b}$ & $1.64 \mathrm{~b}$ & $20.8 \mathrm{cde}$ & $24.0 \mathrm{~h}$ & $24.6 f$ & $8.6 \mathrm{de}$ & $9.2 \mathrm{~b}$ & $7.4 \mathrm{f}$ & $3.14 \mathrm{de}$ & $3.15 \mathrm{~d}$ & $3.23 \mathrm{~d}$ \\
\hline Kozma 194 & $3.5 \mathrm{~d}$ & $4.2 \mathrm{abc}$ & $1.9 \mathrm{bc}$ & $0.91 \mathrm{f}$ & $0.89 \mathrm{k}$ & $0.66 j$ & $23.4 \mathrm{abc}$ & $24.4 \mathrm{~g}$ & $23.9 \mathrm{j}$ & $7.2 \mathrm{fg}$ & $5.9 \mathrm{k}$ & $6.2 \mathrm{i}$ & $3.31 \mathrm{~b}$ & 3.14 & $3.06 \mathrm{j}$ \\
\hline Kozma 2423 & $10.8 \mathrm{abc}$ & $7.1 \mathrm{a}$ & $3.2 \mathrm{abc}$ & $1.20 \mathrm{de}$ & $1.03 \mathrm{~h}$ & $1.06 \mathrm{~h}$ & $20.1 \mathrm{de}$ & $21.2 \mathrm{j}$ & 25.1 & $8.2 \mathrm{ef}$ & $8.9 \mathrm{c}$ & $5.8 \mathrm{j}$ & $3.08 \mathrm{def}$ & $3.07 \mathrm{~h}$ & $3.20 \mathrm{f}$ \\
\hline Significance & $* * *$ & ** & $* * *$ & *** & $* * *$ & *** & $* * *$ & $* * *$ & $* * *$ & $* * *$ & *** & *** & *** & $* * *$ & *** \\
\hline \multicolumn{16}{|c|}{ Red winegrape cultivars } \\
\hline Merlot & $16.0 \mathrm{a}$ & $5.0 \mathrm{c}$ & $1.5 \mathrm{~b}$ & $21 b$ & $0.99 \mathrm{f}$ & 0.98 & $19.9 \mathrm{c}$ & $27.7 \mathrm{a}$ & $25.0 \mathrm{c}$ & $8.2 \mathrm{c}$ & $6.9 \mathrm{e}$ & $8.2 \mathrm{e}$ & $3.15 c$ & $3.13 \mathrm{~d}$ & $3.16 \mathrm{~d}$ \\
\hline Agria & $5.8 \mathrm{~d}$ & $2.7 \mathrm{c}$ & $2.1 b$ & $1.15 b$ & $1.55 b$ & $0.96 \mathrm{f}$ & $19.0 \mathrm{c}$ & $24.0 \mathrm{f}$ & $22.3 \mathrm{~g}$ & $6.2 \mathrm{~d}$ & $5.5 \mathrm{f}$ & $5.5 \mathrm{~g}$ & $3.49 \mathrm{a}$ & $3.58 \mathrm{a}$ & $3.5 \mathrm{la}$ \\
\hline Michurinetz & $9.0 \mathrm{bcd}$ & $10.9 \mathrm{a}$ & $4.6 \mathrm{a}$ & $0.91 \mathrm{c}$ & $1.07 \mathrm{e}$ & $1.01 \mathrm{~d}$ & $23.4 b$ & $22.3 \mathrm{~g}$ & $24.5 \mathrm{~d}$ & $11.9 \mathrm{a}$ & $13.4 \mathrm{a}$ & $10.8 \mathrm{~b}$ & $3.10 \mathrm{c}$ & $2.95 \mathrm{f}$ & $3.10 \mathrm{e}$ \\
\hline Regent & $7.8 \mathrm{~cd}$ & $6.2 \mathrm{bc}$ & $1.2 \mathrm{~b}$ & $1.58 \mathrm{a}$ & $1.68 \mathrm{a}$ & $1.10 \mathrm{c}$ & $22.8 \mathrm{~b}$ & $25.6 \mathrm{~d}$ & $22.4 \mathrm{f}$ & $8.2 \mathrm{c}$ & $7.4 \mathrm{~d}$ & $8.1 \mathrm{f}$ & $3.29 b$ & $3.10 \mathrm{e}$ & $3.22 \mathrm{c}$ \\
\hline Zweigeltrebe & $7.4 \mathrm{~cd}$ & $2.5 \mathrm{c}$ & $1.9 \mathrm{~b}$ & $1.19 b$ & $1.20 \mathrm{~d}$ & $1.11 b$ & $19.2 \mathrm{c}$ & $24.1 \mathrm{e}$ & $24.1 \mathrm{e}$ & $7.7 \mathrm{~d}$ & $7.5 \mathrm{~d}$ & $8.4 \mathrm{~d}$ & $3.15 c$ & $3.14 \mathrm{c}$ & $3.06 \mathrm{f}$ \\
\hline Kozma 55 & $16.3 \mathrm{a}$ & $8.6 \mathrm{ab}$ & $4.3 \mathrm{a}$ & $1.05 b c$ & $0.91 \mathrm{~g}$ & $0.79 \mathrm{~g}$ & $22.4 \mathrm{~b}$ & $27.6 b$ & $25.2 b$ & $10.1 \mathrm{~b}$ & $8.8 \mathrm{~b}$ & $11.3 \mathrm{a}$ & $3.17 \mathrm{c}$ & $3.22 b$ & $3.10 \mathrm{e}$ \\
\hline Kozma 525 & $13.9 \mathrm{ab}$ & $5.8 \mathrm{bc}$ & $1.5 \mathrm{~b}$ & $1.22 \mathrm{~b}$ & $1.34 \mathrm{c}$ & $1.38 \mathrm{a}$ & $20.4 \mathrm{c}$ & $26.8 \mathrm{c}$ & $26.7 \mathrm{a}$ & $9.6 \mathrm{~b}$ & $7.8 \mathrm{c}$ & $9.3 \mathrm{c}$ & $3.11 \mathrm{c}$ & $3.11 \mathrm{e}$ & $3.23 b$ \\
\hline Significance & $* * *$ & $* * *$ & $* * *$ & *** & $* * *$ & *** & $* * *$ & $* * *$ & $* * *$ & $* * *$ & $* * *$ & $* * *$ & $* * *$ & $* * *$ & *** \\
\hline
\end{tabular}

${ }^{\mathrm{z}} 1.0 \mathrm{t} \cdot \mathrm{ha}^{-1}=0.446 \mathrm{ton} /$ acre, $1.00 \mathrm{~g}=0.035 \mathrm{oz}$, titratable acidity is expressed as tartaric acid in $\mathrm{g} \cdot \mathrm{L}^{-1}\left(1.0 \mathrm{~g} \cdot \mathrm{L}^{-1}=1000 \mathrm{ppm}\right)$.

yield not measurable due to vine injury suffered during the previous winter season.

Ns, ${ }^{*},{ }^{* *},{ }^{* * *}$ Nonsignificant or significant at $P \leq 0.05,0.01$, and 0.001 , respectively. Means followed by different letters are significant at $P \leqq 0.05$, Duncan's multiple range test. Where both red and white winegrape cultivars appear within a table subsection, lowercase designations refer to white winegrapes, while uppercase designations refer to red winegrapes. 
fell outside the desirable range; 'Müller-Thurgau' was lowest throughout the trial (Table 3). 'Müller-Thurgau' was additionally lowest in TA in all 3 seasons but all white wine cultivars had TA values within desirable parameters (Table 3). 'Juwel' had lowest $\mathrm{pH}$ in 1993-94, and was the only cultivar that fell outside of the desirable $\mathrm{pH}$ range for winemaking in 1993, suggesting that it may have been harvested somewhat prematurely (Table 3 ). The two red wine cultivars Heroldrebe and Helfensteiner did not differ in terms of yield from the standard 'Spätburgunder' clone 52-86 Fr. except in 1995, but 'Helfensteiner' produced substantially larger berries that approached $2 \mathrm{~g}$ in weight (Table 3 ). This high berry weight could potentially pose an issue for extraction during fermentation, since color, flavor compounds, and phenolics are dependent upon high skin : juice ratios associated with low berry weights. 'Heroldrebe' and 'Helfensteiner' were also lower in SSC than 'Spätburgunder'; 'Helfensteiner' tended towards highest TA and lowest $\mathrm{pH}$ in 2 of 3 years; however, both of the red wine cultivars had berry composition values within desirable parameters (Table 3 ).

Geilweilerhof cultivars. The interspecific hybrid 'Pollux' consistently outyielded standards 'Riesling' and 'Müller-Thurgau' (in 2 of 3 years) throughout the trial (Table 3). 'Castor' also produced high yields as well as highest berry weights in 2 of 3 seasons (Table 3). Selection 'NF 10-126' and 'Orion' produced berries with highest SSC, and these values were somewhat outside the range of desirability; 'Riesling' produced lowest SSC in 2 of 3 seasons (Table 3). Selection 'Gf 111-28$5 \mathrm{l}$ ' consistently produced berries with lowest TA [3.1-4.7 g. $\mathrm{L}^{-1}(3100-4700$ $\mathrm{ppm})]$ and highest $\mathrm{pH}(3.41-3.71)$; these values were somewhat outside the range of commercial acceptability and suggest that this cultivar was not harvested early enough. Highest TA and lowest $\mathrm{pH}$ were consistently found in 'Riesling' and/or 'Müller-Thurgau' berries; these and the values measured on most of the other cultivars were within acceptable ranges for winemaking (Table 3 ).

Geisenheim cultivars. Most selections produced yields greater than or equal to the standard cultivar Riesling. Selections 'Gm 6495-3' 'Gm 643-20', and ' $\mathrm{Gm}$ 646-2' were notable in terms of their overall productivity (Table 3 ), whereas 'Gm 7117-13' and 'Senator' tended to be lowest-yielding. 'Gm 6495-3' also produced the highest berry weight each season (Table 3 ). Most selections produced higher SSC than 'Riesling' each season; some such as 'Senator', 'Gm 7117-13', and 'Gm 6423-12' produced highest SSC, with concentrations exceeding 30\% in 1994 , suggesting that they may have been harvested too late (Table 3). Selections 'Gm 7117-10' and 'Gm 7117-13' produced berries with highest TA, with lowest values occurring each season in 'Senator' (Table 3). 'Gm 7117-10' also tended to have lowest $\mathrm{pH}$ values, with highest values (outside of desirable winemaking range) occurring consistently in 'Senator' (Table 3 ). Red wine selections Gm 6421-15 and 6423-3 produced yields greater than or equal to the 'Pinot noir' standard in 2 of 3 years (Table 3 ). Gm 6421-15 produced highest berry weights each season and 'Pinot noir' the lowest (Table 3). Soluble solids, TA, and $\mathrm{pH}$ values tended to be highest in the 'Pinot noir' berries and Gm 6423-3 had lowest values for SSC ( 2 of 3 years) and TA (Table 3).

Würzburgcultivars. The various cultivars from the Würzburg breeding program did not differ from 'Riesling' or from each other in terms of yield in 1993 or 1995, but 'Ortega' was highest-yielding in 1994 and had highest berry weights throughout (Table 3 ). 'Cantaro' berries had highest SSC in 2 of 3 years and the 1994-95 values were outside the normally acceptable range for white wines, while 'Ortega' had lowest SSC (Table 3). 'Rieslaner' had highest TA in all 3 years, and 'Ortega' had lowest in 2 of 3 seasons (Table 3 ). All TA values were within acceptable parameters. No consistent $\mathrm{pH}$ patterns were noted amongst the cultivars; most $\mathrm{pH}$ values were within acceptable parameters for winemaking.

Hungarian muscat-flavored white wine cultivars. Three of the muscat-flavored cultivars outyielded 'Csabagyongye' in 1993, and 'Zengo' was consistently the highest yielding throughout the 3 years (Table 3 ). Although 'Csabagyongye' has been grown commercially in $\mathrm{BC}$ for many years, severe winter injury in the 199394 winter prevented it from being used as a standard against which to compare the other cultivars in 1994-95. 'Kozma Palne Muscotaly' produced the larg- est berries throughout the course of the trial, while 'Zefir' produced the smallest berries (Table 3). 'Zefir' also had highest SSC in 2 of 3 years and 'Kozma Palne Muscotaly' had lowest percent SSC in the same 2 seasons but this pattern was not apparent in 1995 (Table 3). Soluble solids were within acceptable ranges in 1993-94 for all cultivars, but somewhat high in 1995 (Table 3). 'Zengo' berries had highest TA in 2 of 3 seasons and the lowest $\mathrm{pH}$ throughout the trial; 1993-94 values were somewhat outside acceptable ranges for winemaking, suggesting premature harvest (Table 3). 'Csabagyongye', 'Kozma Palne Muscotaly', and 'Zefir' were usually those cultivars with lowest TA and highest $\mathrm{pH}$; the values of the latter two cultivars were outside acceptable winemaking parameters in 1995 (Table 3).

Hungarian white wine cultivars. Several cultivars and selections were relatively high-yielding, including 'Kozma 195', 'Kozma 2423', 'Bianca', and 'Ezerfurtue', and all outyielded 'Zalagyongye', which has been grown commercially in British Columbia for many years (Table 3). 'Eger 26' consistently produced highest berry weights, while smallest berries occurred in 'Kozma 194' (Table 3). There were no discernible patterns amongst the cultivars with respect to SSC; a few exceeded the upper limits of parameters usually considered acceptable for winemaking (Table 3 ). TA was consistently highest in 'Badacsony 10', while 'Eger 30 ' tended to have highest $\mathrm{pH}$ in 2 of 3 years (Table 3 ).

Miscellaneous red winecultivars. Vitis amurensis hybrid 'Michurinetz' and Hungarian selection 'Kozma 55' were consistently the highest yielding cultivars amongst this group, although they did not outyield the standard, 'Merlot', in 1993 (Table 3). The Hungarian cultivar Agria and Austrian cultivar Zweigeltrebe were lowest yielding in 2 of 3 seasons. The Geilweilerhofintroduction 'Regent' produced largest berries in 2 of 3 seasons, while 'Michurinetz' (1993) and 'Kozma 55' (1994-95) were smallest (Table 3). No discernible and consistent pattern was apparent amongst this group of cultivars in terms of SSC, although 'Agria' and 'Michurinetz' tended to be lowest throughout the trial; a few cultivars exceeded the upper limits of parameters usually considered acceptable for red winemaking in 1994-95 
(Table 3). 'Agria' also produced berries with lowest TA and highest $\mathrm{pH}$, while 'Michurinetz' was consistently high in TA and low in pH (Table 3). All cultivars except 'Michurinetz' consistently produced fruit with acceptable TA and $\mathrm{pH}$ values (Table 3 ).

Must AND WINe COMPosition. Alzey cultivars. 'Faberrebe' was the only Alzey cultivar chosen for winemaking. 'Faberrebe' had higher must SSC, TA, and $\mathrm{pH}$ than standard cultivar Müller-Thurgau, as well as higher wine $\mathrm{pH}$ and ethanol (Table 4).

Freiburg and Weinsberg cultivars. 'Holder', 'Juwel', and 'Nobling' were chosen for winemaking and were compared against standard 'MüllerThurgau'. 'Holder' and 'Nobling' had highest must SSC and 'MüllerThurgau' was lowest (Table 4). 'Müller-Thurgau' was additionally lowest in must and wine TA, while 'Juwel' had highest must and wine TA and lowest must and wine $\mathrm{pH}$ (Table 4). The two red wine cultivars, 'Heroldrebe' and 'Helfensteiner', did not differ from each other in terms of must SSC, ethanol, and wine $\mathrm{pH}$, but were less than the standard 'Spätburgunder' clone 52-86 Fr. (Table 4). 'Helfensteiner' produced substantially higher must TA and wine TA and lower must $\mathrm{pH}$ than both 'Spätburgunder' and 'Heroldrebe' (Table 3). 'Heroldrebe' wines were most intensely colored based upon anthocyanin concentration; the range of anthocyanins increased from $38.2-84.0 \mathrm{mg} \cdot \mathrm{L}^{-1}$ at pressing (Table 4) to $91.9-151.9 \mathrm{mg} \cdot \mathrm{L}^{-1}$ at bottling (data not shown).

Geisenheim cultivars. Preliminary data (BCMAFF, 1992, 1993) suggested that most of the Geisenheim selections in the trial were not appreciably different from the standard cultivar Riesling, or had no unique sensory attributes; thus, only four selections (' $\mathrm{Gm}$ 6497-4', 'Gm 676-2', 'Gm 7116-26', and ' $\mathrm{Gm}$ 7117-10') were retained for winemaking. All selections produced musts greater in SSC than the standard cultivar Riesling, and three of the four had higher ethanol concentration as well (Table 4). Selections 'Gm 6497-4', 'Gm 646-2', and 'Gm 7116-26' had lower must and wine TA and higher must and wine $\mathrm{pH}$ than 'Riesling' (Table 4). The red wine selections 'Gm 6421-15' and 'Gm 6423-3' produced neutral red wines that were no better than 'Pinot noir' standard in preliminary trials (BCMAFF, 1992,
1993); therefore, no red wines were made from any Geisenheim selections in this trial.

Würzburg cultivars. Except for the early-maturing 'Perle', the various cultivars from the Würzburg breeding program produced musts with higher SSC than the 'Riesling' standard (Table 4). 'Cantaro' berries had highest must percent SSC and highest corresponding ethanol in the wine (Table 4). 'Rieslaner' had highest must and wine TA, and lowest must and wine $\mathrm{pH}$, while 'Perle' had lowest must SSC and ethanol, lowest must and wine TA, and highest must and wine $\mathrm{pH}$ (Table 4$)$.

Hungarian muscat-flavored white wine cultivars. Four of the five muscat-flavored cultivars, including standard 'Csabagyongye', were retained for winemaking in 1993. 'Goejseji Zamatos' did not produce sufficient fruit for replicate fermentations. All cultivars had higher must SSC than 'Csabagyongye', but only 'Zefir' had higher corresponding ethanol (Table 4). 'Zengo' was highest in must and wine TA and lowest in must and wine $\mathrm{pH}$ (Table 4). 'Zefir' had lowest wine TA and highest must and wine $\mathrm{pH}$; 'Kozma Palne Muscotaly' and 'Csabagyongye' had lowest must TA (Table 4).

Hungarian white wine cultivars. Amongst the many cultivars and selections that were tested, few demonstrated any unique sensory attributes in preliminary trials (BCMAFF, 1992, 1993). Hence, only 'Bianca' and 'Badacsony 10 ' were retained for winemaking in this trial. The two cultivars did not differ from each other in terms of must SSC and corresponding ethanol, but 'Badacsony 10' had higher must and wine TA, and lower must and wine $\mathrm{pH}$ (Table 4).

Miscellaneousred wine cultivars. All cultivars were equal to or greater than the standard 'Merlot' in must SSC and, 'Michurinetz' excepted, in ethanol (Table 4). 'Michurinetz' was highest amongst this group of cultivars in terms of must SSC, ethanol, and must and wine TA (Table 4). 'Regent' produced musts and wines with lowest TA and highest $\mathrm{pH}$ (Table 4). Both 'Michurinetz' and 'Regent' produced most intensely colored wines; all anthocyanin concentrations in the wines at pressing were higher than 'Merlot' (Table 4). Low ethanol values may have been due to formation of acetic acid or ethyl acetate during the latter stages of primary fermentation, although no volatile acidity was noted by the sensory panel.

SeNSORY ANALYSIS. 'Riesling' alternatives. All the Geisenheim and Würzburg cultivars and selections were tasted as a group to assess their potential as 'Riesling' alternatives. Several of these cultivars were greater than or equal to 'Riesling' in terms of desirable floral, spicy, and citrus aromas (Fig. 1). 'Fontanara' was noteworthy as a cultivar with intense floral and spicy aromas and low intensity of earthy, diesel, and vegetal aromas (Fig. 1). 'Gm 676-2' was distinctive because it had a high hybrid-like nose reminiscent of cooked strawberries. Several cultivars ('Cantaro', 'Rieslaner', 'Gm 676-2', 'Gm 7116-26', and 'Gm 7177-10') were also identified as having intense earthy aromas. The diesel (or "petrol") character often associated with aged 'Riesling' was very prominent in 'Gm 7117-10' (Fig. 1). 'Riesling' was highest in citrus flavor, but the majority of the other cultivars surpassed or equaled 'Riesling' in terms of floral, spicy, peach, and apple flavors (Fig. 2). Four cultivars ('Cantaro', 'Fontanara', 'Gm 676-2', and 'Gm 7116-26' displayed an undesirable "hybrid" note in their retronasal aromas. The diesel characteristic was noticeable in all wines and was particularly prominent in 'Fontanara', 'Gm 7117-10', 'Gm 676-2', and 'Gm 7116-26' (Fig. 2).

PCA showed that spicy, peach, floral, and hybrid aromas and flavors were aligned along $\mathrm{PCl}$ and correlated with each other (Fig. 3). Citrus and apple aroma and flavors, acidity, and tannin were aligned in the opposite direction along $\mathrm{PCl}$, indicating that these attributes were inversely correlated with the aforementioned sensory descriptors. Earthy, diesel, and vegetal aromas and flavors were aligned along PC2 and were therefore unrelated to the other sensory attributes. The two 'Riesling' samples as well as 'Rieslaner' and 'Gm 6497-4' were characterized by citrus, apple, acidity, and tannin (Fig. 3). 'Cantaro', 'Fontanara', 'Gm 676-2', and 'Gm 7116-26' were best characterized by floral, spicy, peach, and hybrid attributes, while 'Gm 7117-10' was described best by vegetal, earthy, and diesel descriptors (Fig. 3).

Müller-Thurgau and alternatives. White wine cultivars from the Weinsberg and Freiburg breeding pro- 
Table 4. Must and wine composition of several winegrape cultivars and selections from European breeding programs, evaluated at Summerland, B.C., in 1993. Cultivars in bold type within each table subsection are commercial standards.

\begin{tabular}{|c|c|c|c|c|c|c|c|}
\hline \multirow[b]{3}{*}{$\begin{array}{l}\text { Origin and } \\
\text { cultivar }\end{array}$} & \multirow{2}{*}{\multicolumn{3}{|c|}{ Must composition }} & \multicolumn{4}{|c|}{ Wine composition } \\
\hline & & & & \multirow[b]{2}{*}{$\begin{array}{c}\text { Ethanol } \\
(\%)\end{array}$} & \multirow[b]{2}{*}{$\begin{array}{l}\text { Titratable } \\
\text { acidity } \\
\left(\mathrm{g} \cdot \mathbf{L}^{-1}\right)^{\mathrm{z}}\end{array}$} & \multirow[b]{2}{*}{$\mathbf{p H}$} & \multirow{2}{*}{$\begin{array}{c}\text { Total } \\
\text { anthocyanins } \\
\text { after press } \\
{\left[\mathbf{m g} \cdot \mathbf{L}^{-1}(\mathbf{p p m})\right]}\end{array}$} \\
\hline & $\begin{array}{c}\text { Soluble } \\
\text { solids (\%) }\end{array}$ & $\begin{array}{c}\text { Titratable } \\
\text { acidity } \\
\left(\mathrm{g} \cdot \mathbf{L}^{-1}\right)^{z}\end{array}$ & $\mathbf{p H}$ & & & & \\
\hline \multicolumn{8}{|c|}{ White winegrape cultivars originating from Landessanstalt fur Rebenzuchtung, Alzey, Germany (Rbeinbessen) } \\
\hline Müller-Thurgau & 17.8 & 6.2 & 3.17 & 12.95 & 6.9 & 2.95 & \\
\hline Faberrebe & 22.9 & 7.0 & 3.29 & 16.48 & 6.5 & 3.25 & \\
\hline Significance & ** & $* *$ & ** & $* * *$ & $* * *$ & $* * *$ & \\
\hline
\end{tabular}

Cultivars originating from Staatliches Weibauinstitut, Freiburg $\left(^{F}\right)$ \& Staatliche Lebr-und Versuchsanstalt, Weinsberg ( $\left.{ }^{W}\right)$, Germany (Baden-Wïrttemburg)

\begin{tabular}{|c|c|c|c|c|c|c|}
\hline & & & & & & \\
\hline Müller-Thurgau & $17.8 \mathrm{c}$ & $6.2 \mathrm{c}$ & $3.17 \mathrm{~b}$ & $12.95 b$ & $6.9 \mathrm{~d}$ & $2.95 b$ \\
\hline Holder $^{W}$ & $20.7 \mathrm{ab}$ & $7.3 \mathrm{bc}$ & $3.24 \mathrm{a}$ & $14.00 \mathrm{a}$ & $7.5 \mathrm{c}$ & $3.08 \mathrm{a}$ \\
\hline Juwel $^{\mathrm{W}}$ & $19.0 \mathrm{bc}$ & $9.0 \mathrm{a}$ & $2.91 \mathrm{c}$ & $12.25 b$ & $8.4 b$ & $2.75 \mathrm{~d}$ \\
\hline Nobling ${ }^{\mathrm{F}}$ & $21.6 \mathrm{a}$ & $7.9 \mathrm{~b}$ & $3.13 b$ & $14.40 \mathrm{a}$ & $8.5 b$ & $2.85 \mathrm{c}$ \\
\hline Significance (white grapes) & $* * *$ & $* * *$ & $* * *$ & $* * *$ & $* * *$ & $* * *$ \\
\hline Spätburgunder 52-86 Fr. & $19.1 \mathrm{~A}$ & $7.6 \mathrm{~A}$ & $3.16 \mathrm{~A}$ & 6.80 & $8.0 \mathrm{C}$ & $3.21 \mathrm{~A}$ \\
\hline Helfensteiner ${ }^{W}$ & $17.9 \mathrm{~B}$ & $8.1 \mathrm{~A}$ & $3.08 \mathrm{~B}$ & 7.00 & $9.9 \mathrm{~A}$ & $3.03 \mathrm{~B}$ \\
\hline Heroldrebe $^{W}$ & $18.1 \mathrm{~B}$ & $6.1 \mathrm{~B}$ & $3.23 \mathrm{~A}$ & 6.65 & $9.0 \mathrm{~B}$ & $3.01 \mathrm{~B}$ \\
\hline Significance (red grapes) & * & $* * *$ & $* * *$ & NS & $* * *$ & $* * *$ \\
\hline \multicolumn{7}{|c|}{ Cultivars originating from Institut fur Rebenzuchtung, Geisenbeim, Germany (Rbeinga } \\
\hline Riesling clone $21 \mathrm{~B}$ Weis & $18.0 \mathrm{c}$ & $9.5 \mathrm{a}$ & $3.03 \mathrm{c}$ & $12.10 \mathrm{c}$ & $8.9 \mathrm{~b}$ & $2.82 \mathrm{~d}$ \\
\hline Gm 6497-4 & $19.4 b$ & $8.0 \mathrm{~b}$ & $3.14 \mathrm{~b}$ & $14.25 \mathrm{~b}$ & $7.9 \mathrm{c}$ & $3.02 \mathrm{c}$ \\
\hline Gm 676-2 & $22.2 \mathrm{a}$ & $7.4 \mathrm{~b}$ & $3.24 \mathrm{a}$ & $15.75 \mathrm{a}$ & $7.2 \mathrm{e}$ & $3.17 \mathrm{a}$ \\
\hline Gm 7117-10 & $20.2 b$ & $10.0 \mathrm{a}$ & $2.99 \mathrm{~d}$ & $12.65 c$ & $9.6 a$ & $2.82 \mathrm{~d}$ \\
\hline Gm 7116-26 & $22.2 \mathrm{a}$ & $7.7 \mathrm{~b}$ & $3.17 \mathrm{~b}$ & $15.80 \mathrm{a}$ & $7.5 \mathrm{~d}$ & $3.07 \mathrm{~b}$ \\
\hline Significance & $* * *$ & $* * *$ & $* * *$ & $* * *$ & $* * *$ & $* * *$ \\
\hline
\end{tabular}

Cultivars originating from Bayerische Landesanstalt fur Wein-, Obst- und Gartenbau, Würzbrg-Veitshochbeim, Germany (Franken)

\begin{tabular}{|c|c|c|c|c|c|c|c|}
\hline \multirow{3}{*}{ Riesling clone 21B Weis } & & \\
\hline & $18.0 \mathrm{~b}$ & $9.5 \mathrm{a}$ & 3.03 & $12.10 \mathrm{c}$ & $8.9 \mathrm{a}$ & & \\
\hline & $23.3 \mathrm{a}$ & $5.7 \mathrm{~b}$ & $3.34 b$ & $16.45 a$ & $5.9 c$ & \multicolumn{2}{|l|}{$3.17 \mathrm{~b}$} \\
\hline Fontanara & $19.7 \mathrm{ab}$ & $6.1 b$ & $3.20 \mathrm{c}$ & $13.80 \mathrm{~b}$ & $6.6 \mathrm{~b}$ & \multicolumn{2}{|l|}{$2.98 \mathrm{c}$} \\
\hline Perle & $17.2 \mathrm{~b}$ & $3.5 \mathrm{c}$ & $3.63 \mathrm{a}$ & $11.75 c$ & $5.0 \mathrm{~d}$ & \multicolumn{2}{|l|}{$3.38 \mathrm{a}$} \\
\hline Significance & * & *** & *** & *** & $* * *$ & \multicolumn{2}{|l|}{$* * *$} \\
\hline \multicolumn{8}{|c|}{ Hungarian muscat-flavored winegrape cultivars } \\
\hline Zefir & $21.4 \mathrm{a}$ & $7.4 \mathrm{~b}$ & $3.41 \mathrm{a}$ & $15.40 \mathrm{a}$ & $5.4 \mathrm{~d}$ & \multicolumn{2}{|l|}{$3.23 \mathrm{a}$} \\
\hline Zengo & $19.3 b$ & $10.3 \mathrm{a}$ & $2.95 \mathrm{~d}$ & $12.20 \mathrm{~b}$ & $10.1 \mathrm{a}$ & \multicolumn{2}{|l|}{$2.78 \mathrm{~d}$} \\
\hline Significance & *** & *** & *** & *** & $* * *$ & \multicolumn{2}{|l|}{$* * *$} \\
\hline \multicolumn{8}{|c|}{ Hungarian white winegrape cultivars and selections } \\
\hline Bianca & 22.0 & 8.2 & 3.26 & 15.70 & 7.3 & \multicolumn{2}{|l|}{3.20} \\
\hline Agria & $19.9 \mathrm{ab}$ & $6.9 \mathrm{~d}$ & $3.37 \mathrm{a}$ & $8.70 \mathrm{~b}$ & $9.9 \mathrm{~d}$ & $3.18 b$ & $325.2 b$ \\
\hline Michurinetz & $23.2 \mathrm{a}$ & $12.5 \mathrm{a}$ & $2.99 \mathrm{c}$ & $9.95 a$ & $12.6 \mathrm{a}$ & $3.17 \mathrm{~b}$ & $651.7 \mathrm{a}$ \\
\hline Regent & $21.9 \mathrm{ab}$ & $5.5 \mathrm{e}$ & $3.40 \mathrm{a}$ & $8.70 \mathrm{~b}$ & $8.6 \mathrm{e}$ & $3.33 \mathrm{a}$ & $630.3 \mathrm{a}$ \\
\hline Zweigeltrebe & $18.9 b$ & $6.4 \mathrm{~d}$ & $3.14 b$ & $6.95 \mathrm{~d}$ & $9.6 \mathrm{~d}$ & $2.87 \mathrm{e}$ & $177.5 \mathrm{c}$ \\
\hline Kozma 55 & $22.4 \mathrm{ab}$ & $9.5 b$ & $3.15 b$ & $8.00 \mathrm{c}$ & $11.4 \mathrm{~b}$ & $3.11 \mathrm{c}$ & $143.0 \mathrm{~cd}$ \\
\hline Kozma 525 & $20.0 \mathrm{ab}$ & $8.2 \mathrm{c}$ & $3.11 \mathrm{~b}$ & $7.20 \mathrm{~d}$ & $11.0 \mathrm{~b}$ & $2.99 \mathrm{~d}$ & $323.0 \mathrm{~b}$ \\
\hline Significance & * & $* * *$ & $* * *$ & $* * *$ & $* * *$ & $* * *$ & $* * *$ \\
\hline
\end{tabular}




\section{VARIETY TRIALS}
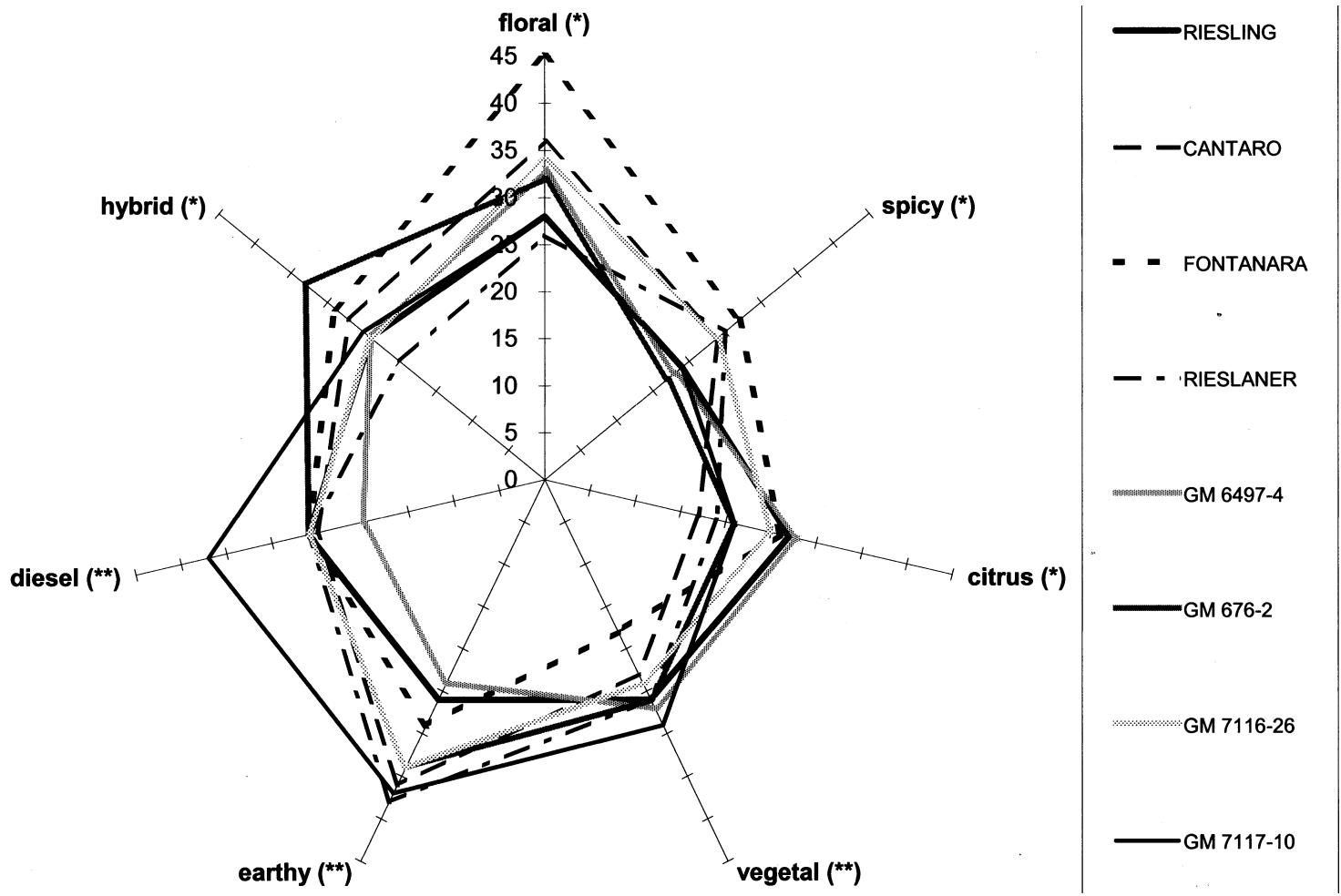

Fig. 1. Mean sensory profile of aroma descriptors for 1993 British Columbia wines produced from eight 'Riesling' alternative cultivars. ${ }^{\mathrm{NS},}{ }^{*},{ }^{* *},{ }^{* * *}$ Nonsignificant or significant at $P \leq \mathbf{0 . 0 5}, 0.01$, or 0.001 , respectively.
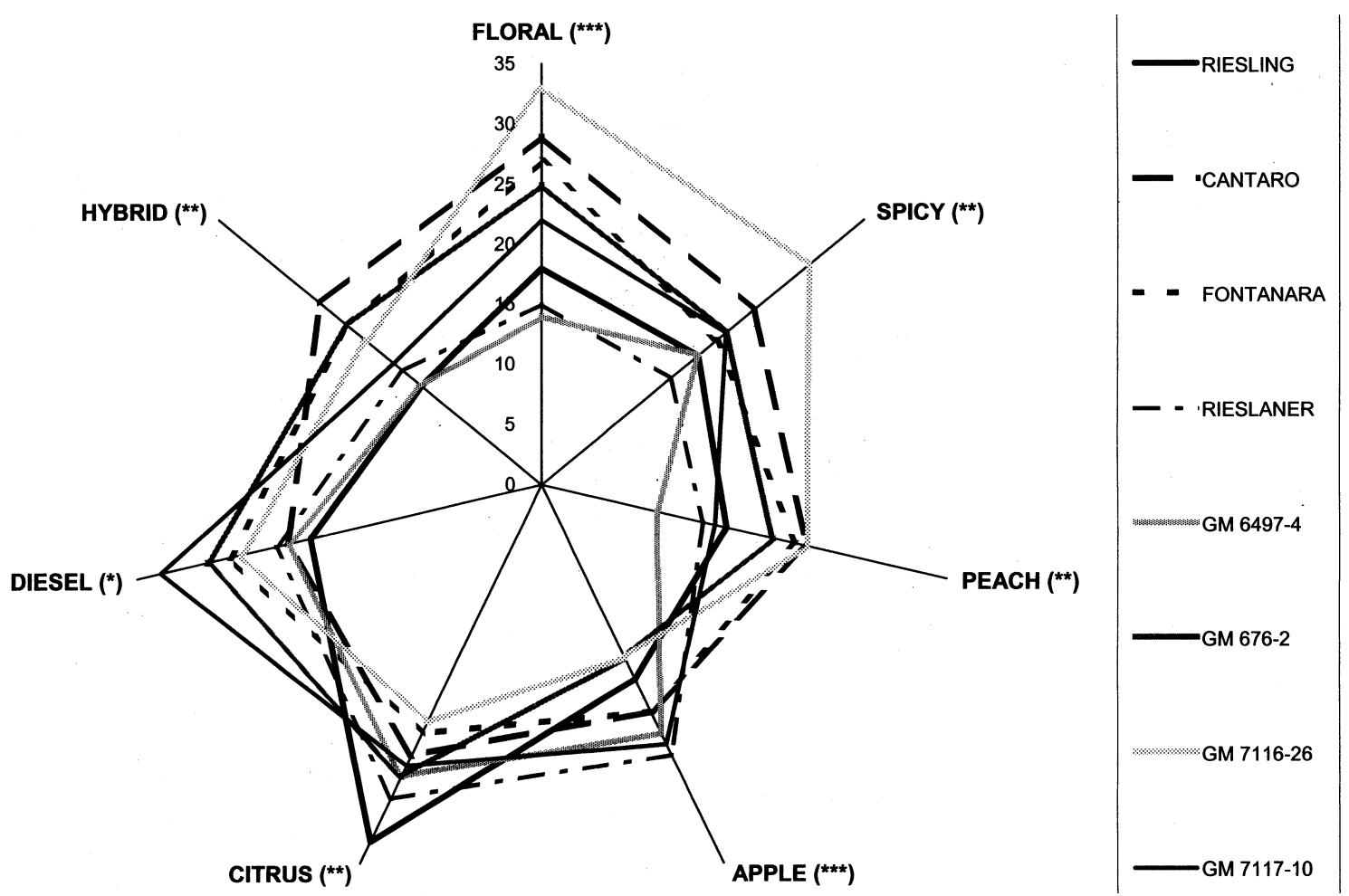

Fig. 2. Mean sensory profile of flavor descriptors for 1993 British Columbia wines produced from eight 'Riesling' alterna-

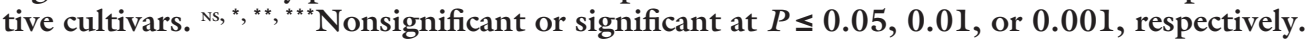




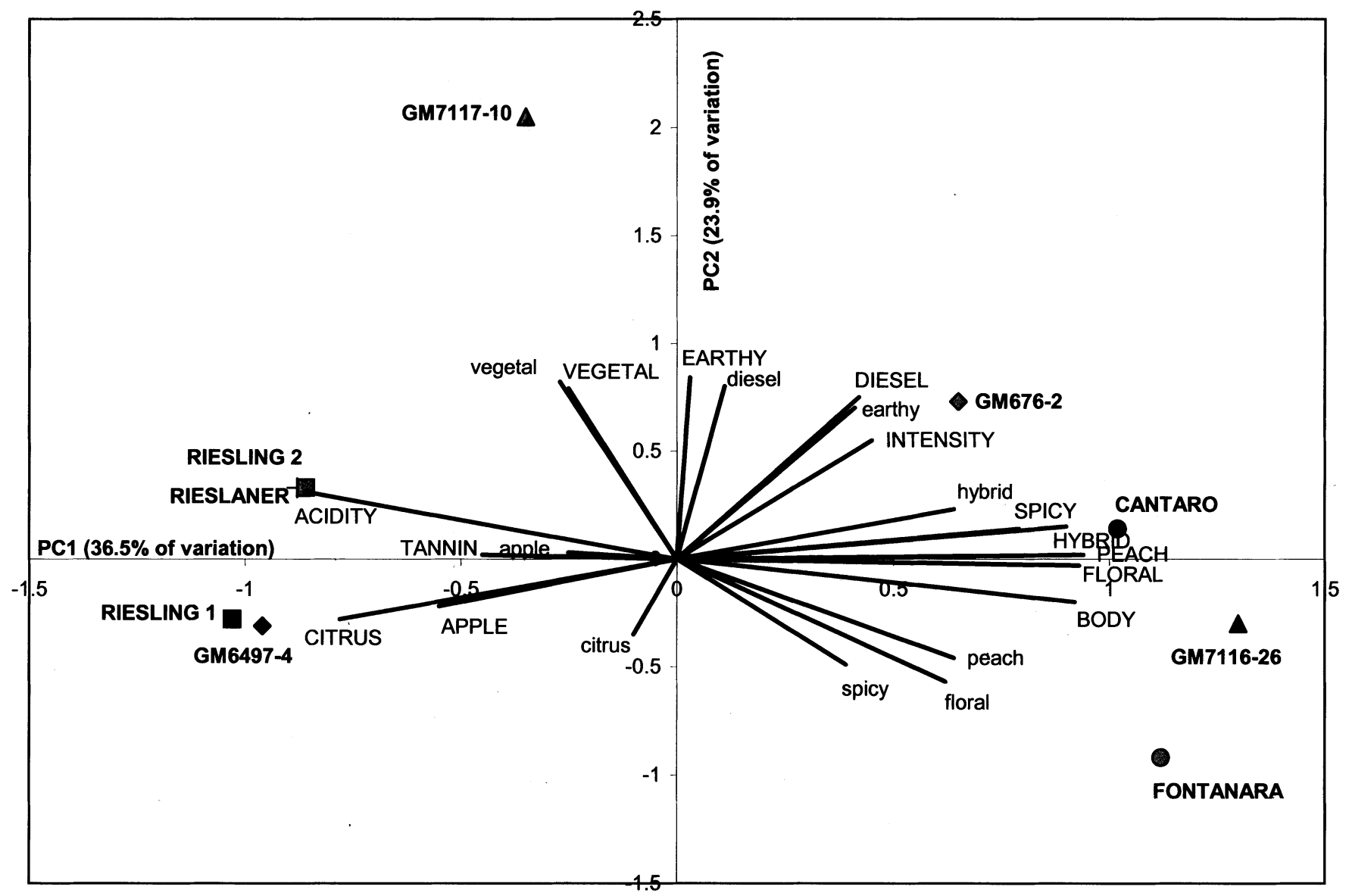

Fig. 3. Projection of wine aroma (lowercase) and flavor (uppercase) data from seven 'Riesling' alternative cultivars on principal components 1 (36.9\% of variability) and 2 (23.9\% of variability).

grams, plus 'Perle' from the Würzburg program, were included in this tasting. Most cultivars exceeded or equaled 'Müller-Thurgau' with respect to all aroma and flavor descriptors (Fig. 4). 'Faberrebe' was notable in terms of high intensity of floral/muscat, apple/ pear, and fig / raisin aromas and flavors, as well as spicy flavor. 'Holder' was also noteworthy in terms of intensity of floral/muscat and apple/pear aromas. Other cultivars tended to display very similar aroma and flavor profiles (Fig. 4).

Hungarian white wine cultivars. The four muscat-flavored cultivars ('Csabagyongye', 'Kozma Palne Muscotaly', 'Zefir', and 'Zengo') plus 'Bianca' and 'Badacsony 10 ' were selected for this tasting. All cultivars exceeded or equaled the standard 'Csabagyongye' in terms of all aroma and flavor attributes. 'Kozma Palne Muscotaly' and 'Zefir' were particularly notable in terms of their high intensity of muscat and apricot/peach aromas, while 'Zefir' also displayed high muscat, cooked strawberry, and vegetal flavors (Fig. 5). Both 'Zengo' and 'Badacsony 10 ' were noteworthy in terms of high apricot/peach flavor.

Pinot noir-like cultivars. 'Spätburgunder' $52-86 \mathrm{Fr}$. was included as a standard in this tasting, along with 'Frühburgunder', 'Helfensteiner', 'Heroldrebe', and 'Samtröt'. Field performance of 'Frühburgunder' and 'Samtröt' are reported elsewhere (Reynolds, 1996a). All cultivars, with the exception of 'Frühburgunder', exceeded or equaled 'Spätburgunder' in terms of all aroma and flavor descriptors (Figs. 6, 7). Aroma profiles of the five cultivars were remarkably similar, however, and 'Frühburgunder' and 'Helfensteiner' were considerably less grassy and vegetal than the others (Fig 6). An aroma attribute described by the tasters as "animal-like" appeared to be less apparent in 'Frühburgunder' than the others. This confirms data from the 1995 tasting of the 'Pinot noir' clones in which 'Frühburgunder' was included; it tended to be the least intense in all aroma and flavor descriptors compared to the other 'Pinot noir' clones (Reynolds, 1996a). 'Spätburgunder' had a tendency to be least intense in terms of cherry, berry, and currant aromas (Fig. 6). 'Helfensteiner' appeared noteworthy in terms of cherry, berry, and currant flavors, while 'Samtröt' was the most intensely colored wine, with the highest acidity and body, and most intense finish (Fig. 7).

Miscellaneous red cultivars. All cultivars exceeded or equaled the standard 'Merlot' in all aroma and flavor attributes (Figs. 8, 9). Noteworthy cultivars included 'Regent', with intense cherry and spicy aroma attributes, and both 'Michurinetz' and 'Kozma 55', with high currant and plum aromas (Fig. 8). The six cultivars had very similar flavor profiles (Fig. 9) but 'Michurinetz' was notable in terms of high berry and spicy flavors, body, and color. The standard 'Merlot' appeared to be least intense in the fruit flavors and color but most vegetal in flavor (Fig. 9). 


\section{VARIETY TRIALS}

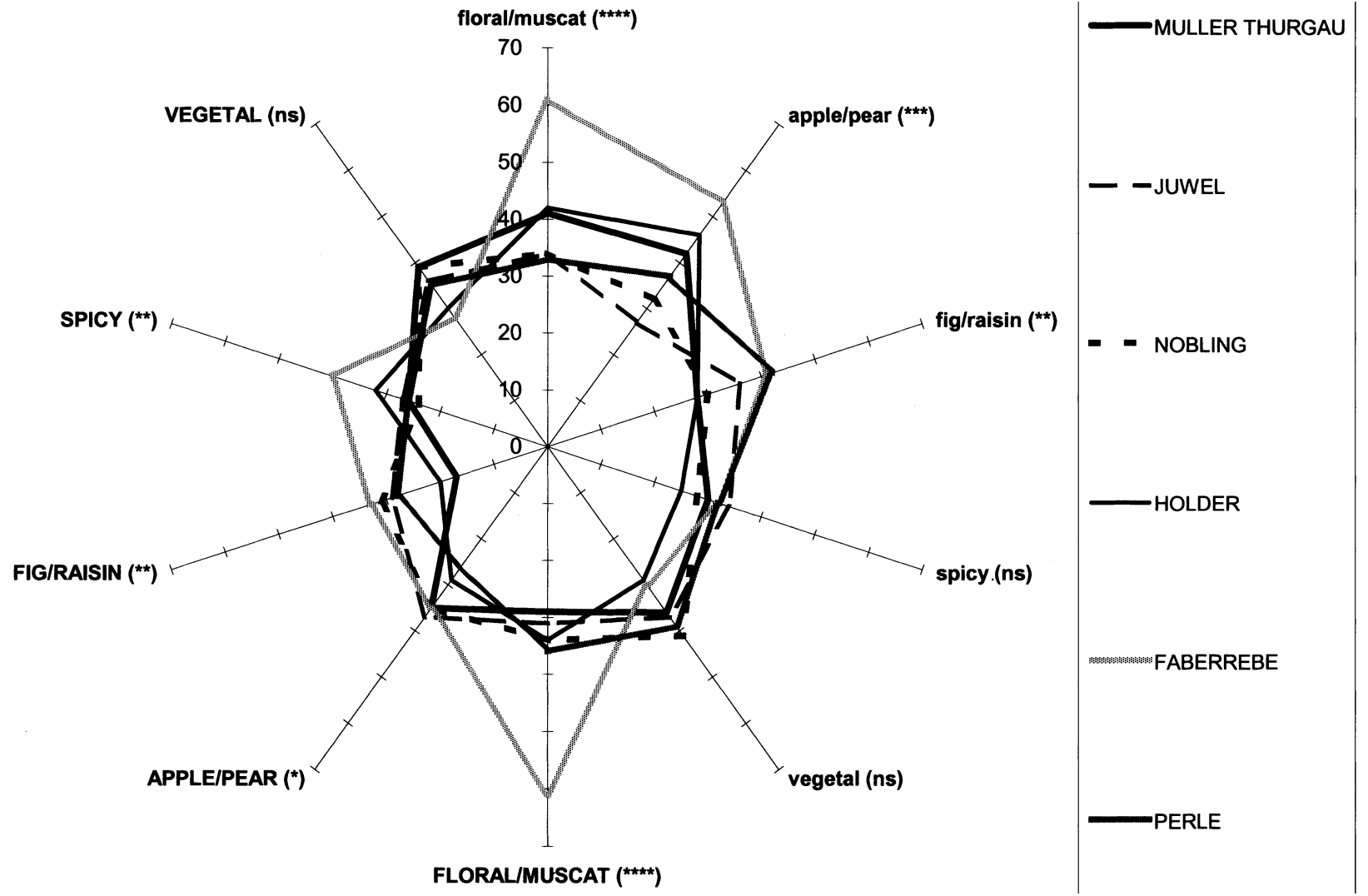

Fig. 4. Mean sensory profile of aroma and flavor descriptors for 1993 British Columbia wines produced from several 'Müiler-Thurgau' alternative cultivars. ${ }^{\mathrm{Ns},}{ }^{*}{ }^{* *},{ }^{* *}$ Nonsignificant or significant at $P \leq 0.05,0.01$, or 0.001 , respectively.

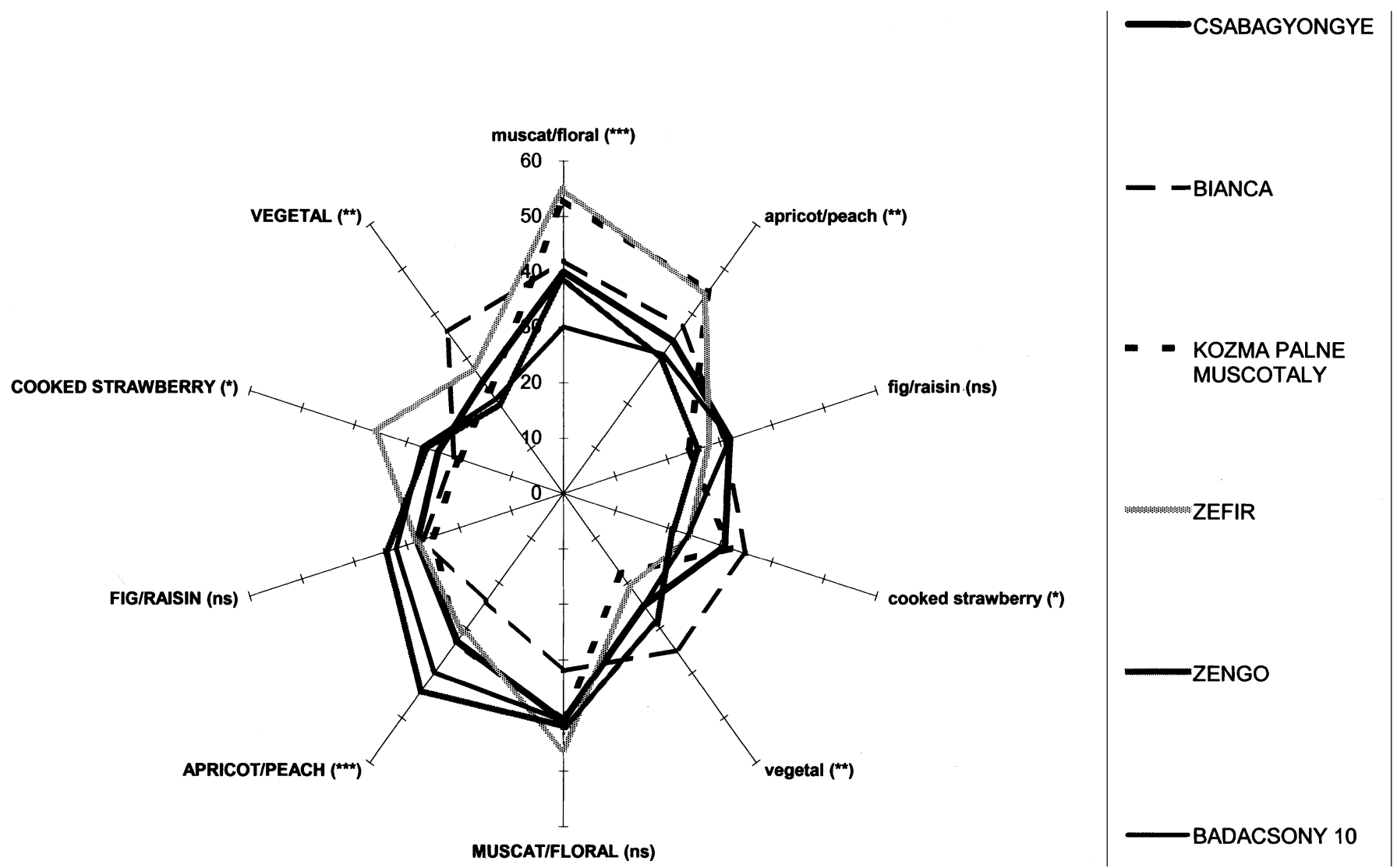

Fig. 5. Mean sensory profile of aroma and flavor descriptors for 1993 British Columbia wines produced from several Hun-

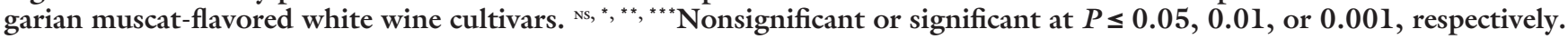



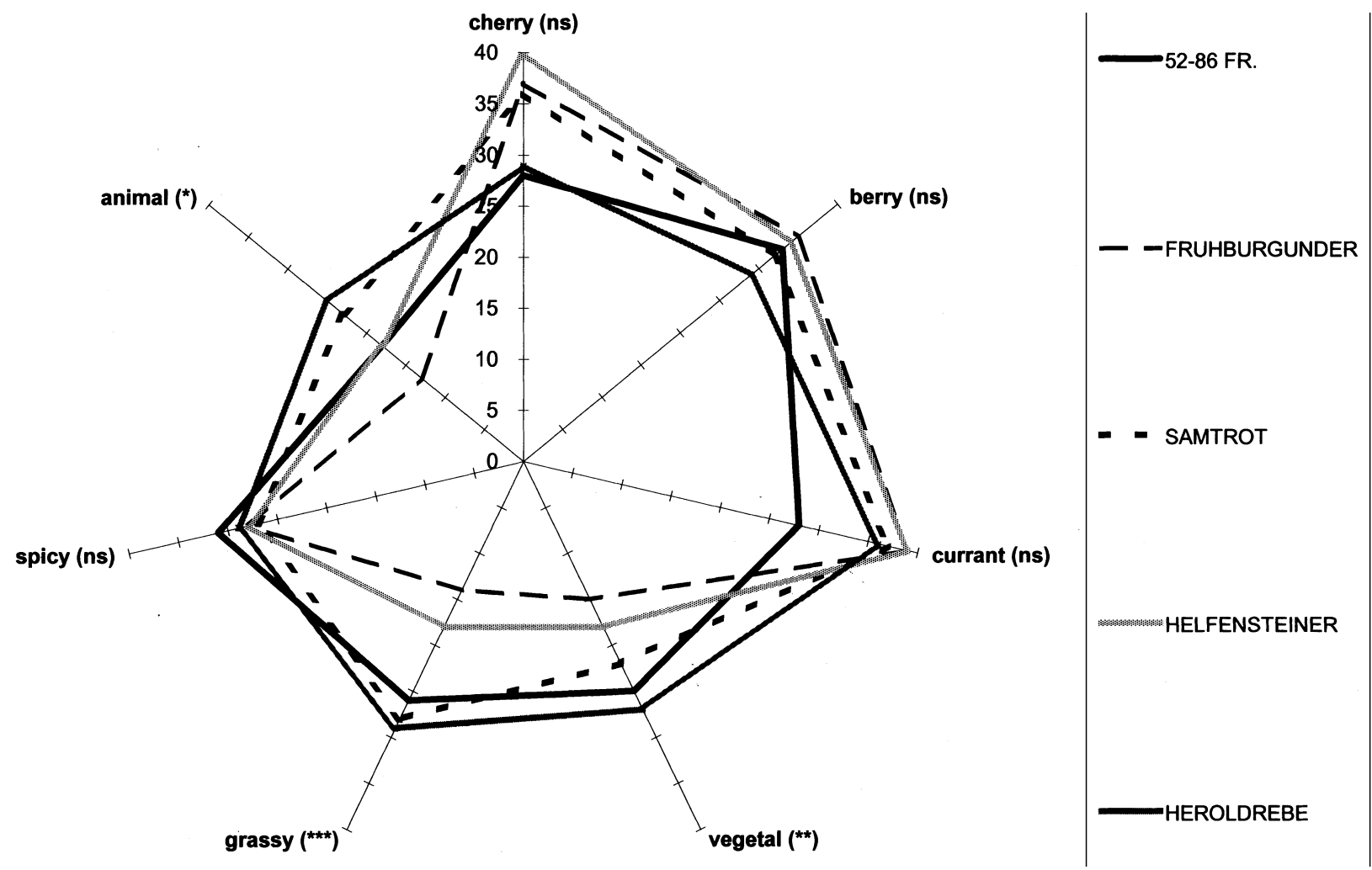

Fig. 6. Mean sensory profile of aroma descriptors for 1993 British Columbia wines produced from several 'Pinot noir'-like cultivars. ${ }^{\mathrm{N},},{ }^{*}{ }^{* *},{ }^{* * *}$ Nonsignificant or significant at $P \leq 0.05,0.01$, or 0.001 , respectively.
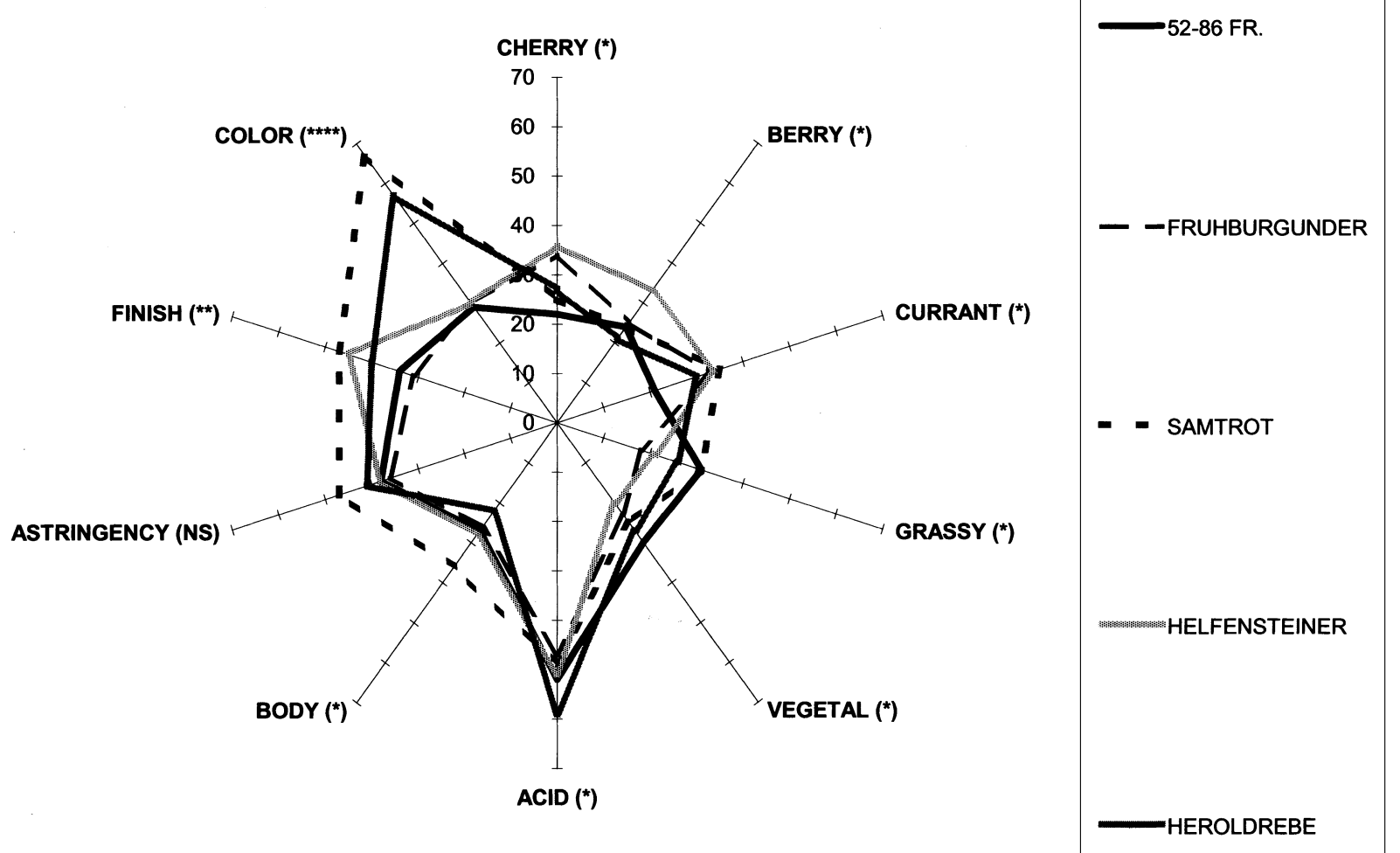

Fig. 7. Mean sensory profile of flavor descriptors for 1993 British Columbia wines produced from several 'Pinot noir'-like cultivars. ${ }^{\mathrm{Ns},}{ }^{*},{ }^{* *},{ }^{* *}$ Nonsignificant or significant at $P \leq 0.05,0.01$, or 0.001 , respectively. 

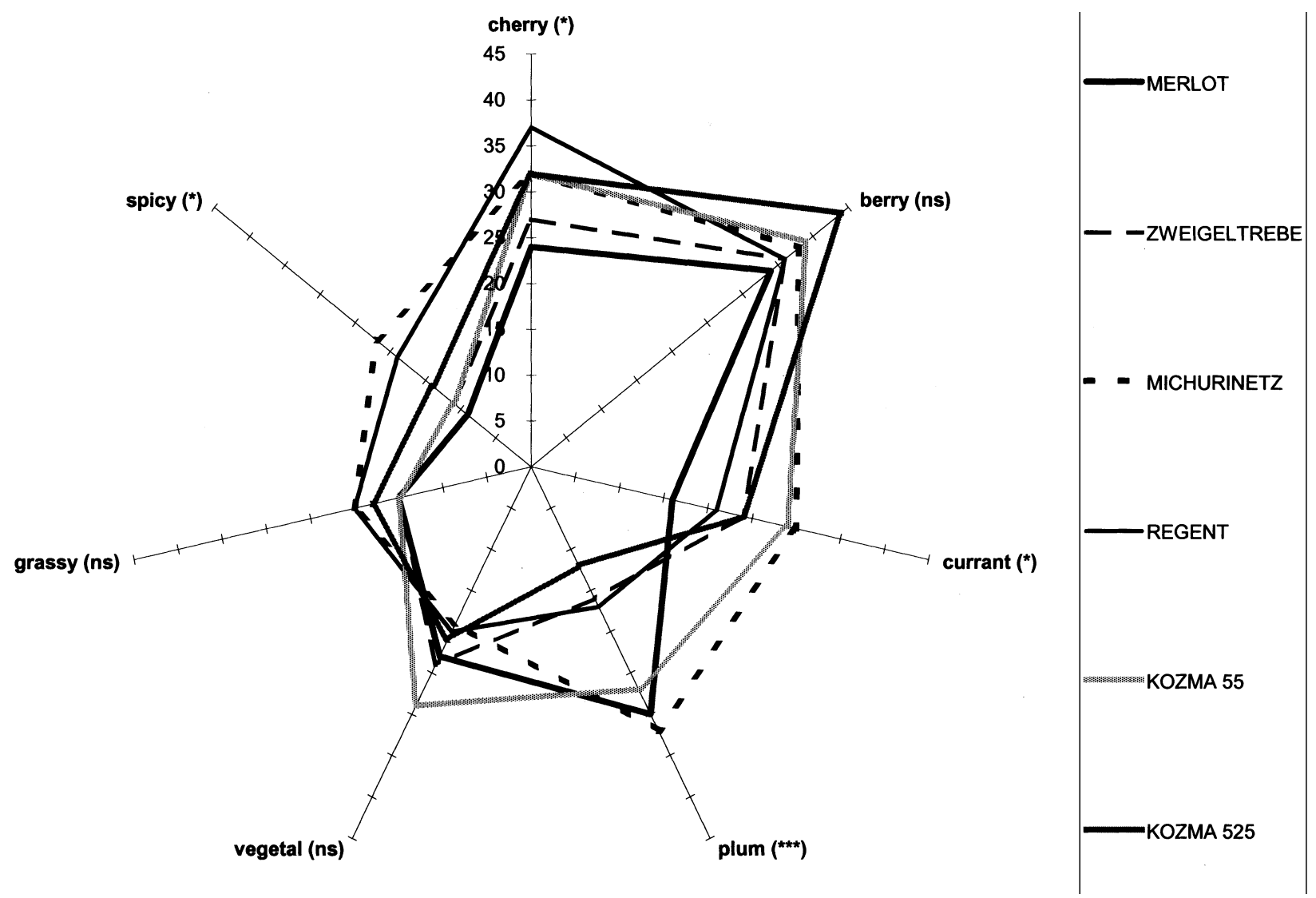

Fig. 8. Mean sensory profile of aroma descriptors for 1993 British Columbia wines produced from seven miscellaneous red wine cultivars. ${ }^{\mathrm{Ns},}{ }^{*}{ }^{* *},{ }^{* \star *}$ Nonsignificant or significant at $P \leq 0.05,0.01$, or 0.001 , respectively.

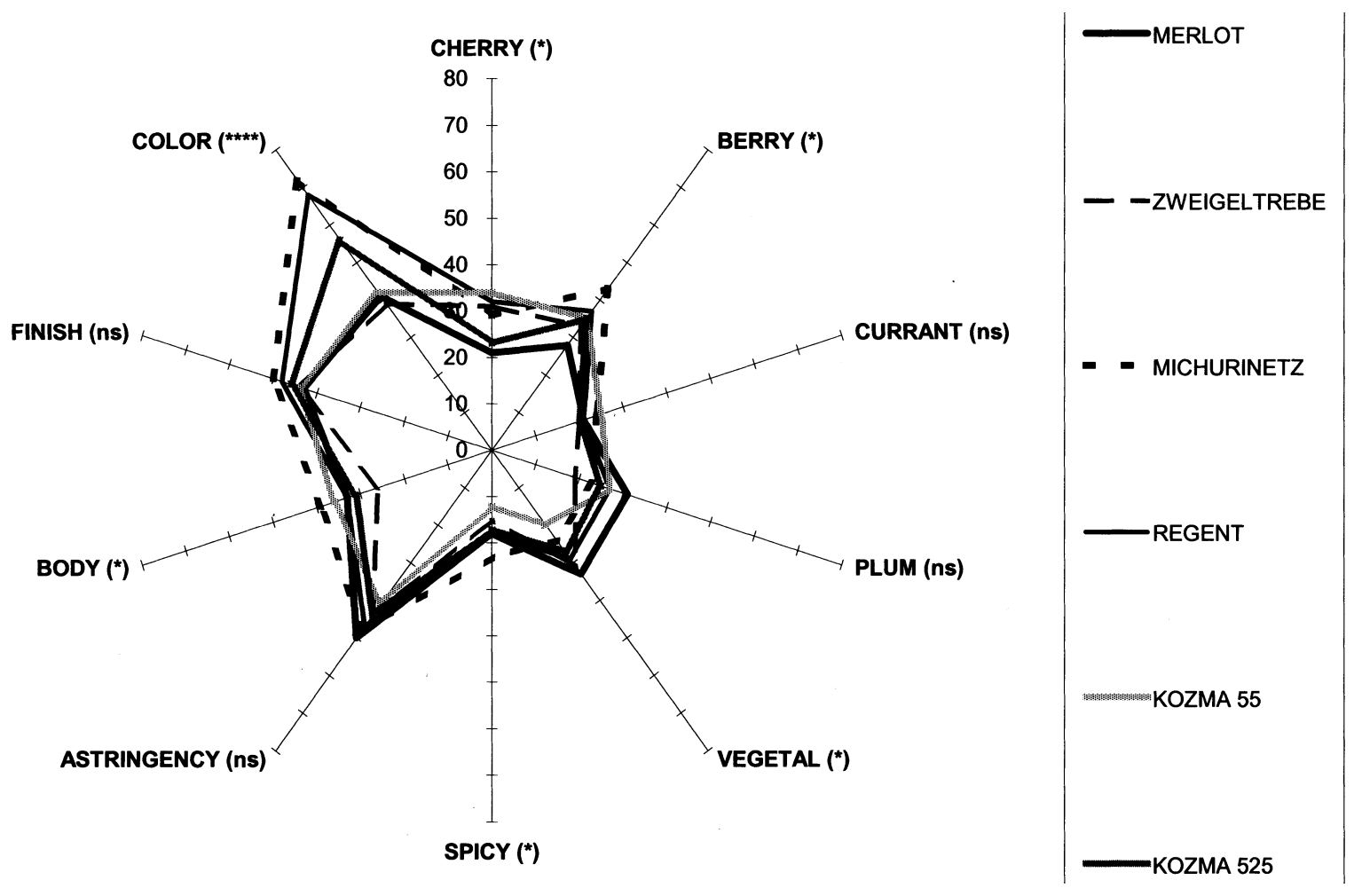

Fig. 9. Mean sensory profile of flavor descriptors for 1993 British Columbia wines produced from seven miscellaneous red wine cultivars. ${ }^{\mathrm{Ns}, ~}{ }^{* * *}{ }^{* * *}$ Nonsignificant or significant at $P \leq 0.05,0.01$, or 0.001 , respectively. 


\section{Conclusions and grower benefits}

The information compiled in this study and in the reports from the preliminary trial (BCMAFF, 1992, 1993) provides a comprehensive set of data that may benefit growers throughout North America. It has particular applicability to those regions in which $V$. vinifera cannot be grown, where latematuring cultivars cannot consistently mature their fruit, and for growers with interest in growing grapes in the absence of pesticides. It also appears to be the first evaluation of most of these cultivars and selections in North America. The preliminary trial (BCMAFF, 1992, 1993) suggested that 37 of the original 85 cultivars included in the evaluation had commercial potential. These included five 'Chardonnay' clones and six 'Pinot noir' clones that are described elsewhere (Reynolds, 1996). Also among the original 37 were cultivars that have become established in the industry ('Auxerrois', 'Csabagyongye', 'Ehrenfelser', 'Merlot', 'Müller-Thurgau', 'Ortega', 'Schönburger', and 'Weissburgunder'). However, several other cultivars emerged from this trial with excellent potential from both a horticultural and commercial winemaking point of view. White wine cultivars considered noteworthy included several from Geisenheim ('Gm 6497-4', 'Gm 676-2', 'Gm 7116-26', and 'Gm 7117-10'), Weinsberg ('Juwel'), Würzburg ('Cantaro', 'Fontanara', and 'Rieslaner'), and Hungary ('Bianca', 'Badacsony 10', 'Kozma Palne Muscotaly', 'Zefir', and 'Zengo'). Red wine cultivars with potential were 'Pinot noir' alternatives 'Heroldrebe' and Helfensteiner', and the more deeply pigmented cultivars 'Regent', 'Michurinetz', 'Kozma 55', and 'Kozma 525'.

This study was also somewhat unique in its use of organic management techniques. Although disease incidence data were not collected, silicate sprays nonetheless adequately controlled powdery mildew, while oil and detergent provided acceptable control for leafhoppers. A major challenge was nitrogen management; the lack of sufficient quantity of organic nitrogen sources caused reductions in vine vigor and yields over the 3 years of this trial to a point where yields of some cultivars were noneconomic.
Optimization of nitrogen input was also cited as a major issue in organic management of vineyards in Italy (Malusa et al., 2004; Marenghi, 2002); however, when organic nitrogen fertilizer was applied in adequate amounts [300 $\mathrm{kg} \cdot \mathrm{ha}^{-1}(267.7 \mathrm{lb} /$ acre $)$ at $11 \%$ actual N], yields were not reduced, and berry polyphenols were increased over those from conventionally managed treatments. Growers considering organic viticulture are therefore urged to ensure that a consistent supply of organic nitrogen is available to sustain vine vigor and economic yields.

\section{Literature cited}

Alleweldt, G. 1980. The breeding of fungus- and phylloxera-resistant grapevine varieties, p. 242-250. In: H.P. Olmo (ed.). Proc. 3rd Intl. Symp. Grape Breeding, Univ. of California Press, Davis.

Alleweldt, G. and E. Dettweiler-Münch. 1992. The genetic resources of Vitis. Genetic and geographic origin of grape cultivars, their prime names and synonyms. Institut für Rebenzüchtung Geilweilerhof, Germany.

Amerine, M.A. and C.S. Ough. 1980. Methods for the analysis of musts and wines. Wiley, New York.

Barillère, J.M., A. Collas, C. Bougerey, and C. Palgé. 1995. Clonal selection in Champagne, p. 33-39. In: J. Rantz (ed.). Proc. Intl. Symp. Clonal Selection, Amer. Soc. Enol. Viticult., Davis, Calif.

Becker, H. 1984. White grape varieties for cool climate, p. 46-62. In: D.A. Heatherbell, P.B. Lombard, F.W. Bodyfelt, and S.F. Price (eds.). Proc. 1st Intl. Symp. Cool Climate Viticult. Enol., Oregon State Univ. Tech. Publ. 7628.

Becker, N. 1988. Performance of Pinot noir clones, p. 282-284. In: R.E. Smart, R. Thornton, S. Rodriguez, and J. Young (eds.). Proc. 2nd Intl. Symp. Cool Climate Viticult. and Oenology, N.Z. Soc. Viticult. Oenology, Auckland.

Bernard, R. and M. Leguay. 1984. Clonal variability of Pinot noir in Burgundy and its potential adaptation under other cooler climates, p. 63-74. In: D.A. Heatherbell, D.A., P.B. Lombard, F.W. Bodyfelt, and S.F. Price (eds.). Proc. 1st Intl. Symp. Cool Climate Viticult. Enol., Oregon State Univ. Tech. Publ. 7628 .

Bettiga, L.J. 1995. Comparison of Merlot and Chardonnay clones in Monterey County: A preliminary report, p. 93-95. In: J. Rantz (ed.). Proc. Intl. Symp. Clonal Selection, Amer. Soc. Enol. Viticult., Davis, Calif.
Boidron, R. 1995. Clonal selection in France. Methods, organization, and use, p. 1-7. In: J. Rantz (ed.). Proc. Int. Symp. Clonal Selection, Amer. Soc. Enol. Viticult., Davis, Calif.

British Columbia Ministry of Agriculture, Fisheries, and Food. 1992. Evaluation of European grape varieties. Summary Rpt. 1987-92. B.C. Min. Agr. Fish. Food, AgriFood Reg. Subsidiary Agreement.

British Columbia Ministry of Agriculture, Fisheries, and Food. 1993. Evaluation of European grape varieties. Suppl. Summary Rpt. 1987-92. B.C. Min. Agr. Fish. Food, Agri-Food Reg. Subsidiary Agreement.

British Columbia Ministry of Agriculture, Fisheries, and Food. 1996. Grape production guide. B.C. Min. Agr. Fish. Food, Ext. Systems Branch, Victoria, B.C.

Certified Organic Association of British Columbia. 2003. British Columbia certified organic production operation policies and management standards. Ver. 5. Book 2. Certified organic management standards. COABC, Vernon, B.C.

Drought, B.G. and B. Stretch. 1996. Weather observations for 1995. Summaries and averages for 80 years of continuous recording. Misc. Publ., Agr. Agri-Food Canada, Res. Sta., Summerland, B.C.

Hillebrand, W., H. Lott, and F. Pfaff. 1984. Taschenbuch der Rebsorten. Fachverlag Dr. Fraund GmbH.

Kelley, C.C. and R.H. Spilsbury. 1949. Soil survey of the Okanagan and Similkameen Valleys, British Columbia. Rpt. No. 3, B.C. Survey, B.C. Dept. Agr., Victoria.

Malusa, E., E. Laurenti, E. Ghibaudi, and L. Rolle. 2004. Influence of organic and conventional management on yield and composition of grape cv. Grignolino. Acta Hort. (In press.)

Marenghi, M. 2002. Biologico, una questione di cultura. Vignevini 6:51-53.

Noble, A.C. 1988. Analysis of wine sensory properties, p. 10-28. In: H. Linskens and J. Jackson (eds.). Modern methods of plant analysis. Wine analysis, Vol. 6. SpringerVerlag, Berlin.

Pool, R.M., T. Henick-Kling, G.E. Howard, B.K. Gavitt, and T.J. Johnson. 1995. Pinot noir clonal research in New York, p. 45-51. In: J. Rantz (ed.). Proc. Intl Symp. Clonal Selection, Amer. Soc. Enol. Viticult., Davis, Calif.

Price, S.F., P.B. Lombard, and B.TWatson. 1988. Pinot noir clones and their effects on cluster morphology and grape composition, p. 279-281. In: R.E. Smart, R. Thornton, S. Rodriguez, and J. Young (eds.). Proc. 2nd Intl. Symp. Cool Climate Viticult. 
Oenology, N.Z. Soc. Viticult. Oenology, Auckland.

Price, S.F. and B.T Watson. 1995. Preliminary results from an Oregon Pinot noir clonal trial, p. 40-44. In: J. Rantz (ed.). Proc. Intl. Symp. Clonal Selection, Amer. Soc. Enol. Viticult., Davis, Calif.

Reynolds, A.G. 1996a. Einfluss von Klonen auf die Sensorik von Spatburgunder und Chardonnay. Die Winzer Zeitschrift $11(4): 28-30$.

Reynolds, A.G. 1996b. Alternative control measures for powdery mildew. Vineyard Winery Mgt. 22(3):25-26,28,42-43,46.

Reynolds, A.G., P.L. Sholberg, D.A. Wardle, and P. Haag. 1996. Use of potassium silicate for the control of powdery mildew (Uncinula necator) in Vitisvinifera cultivar Bacchus. Amer. J. Enol. Viticult. 47:421-428.

Watson, B.T., P.B. Lombard, S.F. Price, M. McDaniel, and D. Heatherbell. 1988. Evaluation of Pinot noir clones in Oregon, p. 276-278. In: R.E. Smart, R. Thornton, S. Rodriguez, and J. Young (eds.). Proc. 2nd Intl. Symp. Cool Climate Viticult. Oenology, N.Z. Soc. Viticult. Oenology, Auckland.

Wolf, T.K. and M.K. Miller. 2001. Crop yield, fruit quality, and winter injury of 12 red-fruited wine grape cultivars in Northern Virginia. J. Amer. Pomol. Soc. 55:241-250.

Wolf, T.K. and M.K. Warren. 2000. Crop yield, grape quality, and winter injury of eight red-fruited wine grape cultivars in Northern Virginia. J. Amer. Pomol. Soc. $54: 34-43$.

Wolpert, J.A. 1995. Evaluation of winegrape clones in coastal California: current activities and future prospects, p. 74-80. In: J. Rantz (ed.). Proc. Intl. Symp. Clonal Selection, Amer. Soc. Enol. Viticult., Davis, Calif. 